\title{
High transmissibility and fetal pathogenicity of recent Zika virus strains from the African lineage
}

Fabien Aubry ${ }^{1 \ddagger}$, Sofie Jacobs ${ }^{2 \ddagger}$, Maïlis Darmuzey ${ }^{3 \ddagger}$, Sebastian Lequime ${ }^{4,5}$, Leen Delang $^{2}$, Albin Fontaine $6,7,8$, Natapong Jupatanakul ${ }^{1,9}$, Elliott F. Miot ${ }^{1}$, Stéphanie Dabo $^{1}$, Caroline Manet $^{10}$, Xavier Montagutelli ${ }^{10}$, Artem Baidaliuk ${ }^{1,11}$, Fabiana Gambaro $^{11}$, Etienne Simon-Lorière ${ }^{11}$, Maxime Gilsoul ${ }^{3}$, Claudia M. Romero-Vivas ${ }^{12}$, Van-Mai Cao-Lormeau ${ }^{13}$, Richard G. Jarman ${ }^{14}$, Cheikh T. Diagne ${ }^{15}$, Oumar Faye ${ }^{15}$, Ousmane Faye ${ }^{15}$, Amadou A. Sall ${ }^{15}$, Johan Neyts ${ }^{2}$, Laurent Nguyen ${ }^{3}$, Suzanne J. F. Kaptein $^{2 \dagger *}$, Louis Lambrechts ${ }^{1 \dagger *}$

${ }^{1}$ Insect-Virus Interactions Unit, Institut Pasteur, CNRS UMR2000, Paris, France ${ }^{2} \mathrm{KU}$ Leuven Department of Microbiology, Immunology and Transplantation, Rega Institute for Medical Research, Laboratory of Virology and Chemotherapy, Leuven, Belgium

${ }^{3}$ GIGA-Stem Cells / GIGA-Neurosciences, Interdisciplinary Cluster for Applied Genoproteomics (GIGA-R), University of Liège, C.H.U. Sart Tilman, Liège, Belgium ${ }^{4} \mathrm{KU}$ Leuven Department of Microbiology, Immunology and Transplantation, Rega Institute, Laboratory of Clinical and Epidemiological Virology, Leuven, Belgium ${ }^{5}$ Cluster of Microbial Ecology, Groningen Institute for Evolutionary Life Sciences, University of Groningen, The Netherlands

'Unité Parasitologie et Entomologie, Département Microbiologie et Maladies Infectieuses, Institut de Recherche Biomédicale des Armées (IRBA), Marseille, France ${ }^{7}$ Aix Marseille University, IRD, SSA, AP-HM, UMR Vecteurs - Infections Tropicales et Méditerranéennes (VITROME), Marseille, France

${ }^{8}$ IHU Méditerranée Infection, Marseille, France

${ }^{9}$ National Center for Genetic Engineering and Biotechnology (BIOTEC), Pathum Thani, Thailand

${ }^{10}$ Mouse Genetics Laboratory, Institut Pasteur, Paris, France

${ }^{11}$ Evolutionary Genomics of RNA Viruses Group, Institut Pasteur, Paris, France

${ }^{12}$ Laboratorio de Enfermedades Tropicales, Departamento de Medicina, Fundación Universidad del Norte, Barranquilla, Colombia

${ }^{13}$ Institut Louis Malardé, Papeete, Tahiti, French Polynesia 
${ }^{14}$ Viral Diseases Branch, Walter Reed Army Institute of Research, Silver Spring, Maryland, United States of America

${ }^{15}$ Institut Pasteur Dakar, Arbovirus and Viral Hemorrhagic Fevers Unit, Senegal

¥These authors contributed equally

tThese authors contributed equally

${ }^{*}$ Correspondence to Suzanne J. F. Kaptein (email: suzanne.kaptein@kuleuven.be) and Louis Lambrechts (email: louis.lambrechts@pasteur.fr)

\section{Summary}

The global emergence of Zika virus (ZIKV) in the last decade revealed the unprecedented ability for a mosquito-borne virus to cause congenital birth defects such as microcephaly. A puzzling aspect of ZIKV emergence is that all human outbreaks and birth defects to date have been exclusively associated with the Asian ZIKV lineage, despite a growing body of laboratory evidence pointing towards higher transmissibility and pathogenicity of the African ZIKV lineage. Whether this apparent paradox reflects the use of relatively old African ZIKV strains in most laboratory studies is unclear. Here, we experimentally compared the transmissibility and pathogenicity of seven lowpassage ZIKV strains representing the recently circulating viral genetic diversity. We found that recent African ZIKV strains largely outperformed their Asian counterparts in mosquito transmission kinetics experiments, which translated into a markedly higher epidemic potential in outbreak computer simulations. In addition, African ZIKV strains were significantly more lethal than Asian ZIKV strains in immunocompromised adult mice. Finally, prenatal infection of immunocompetent mouse embryos with an African ZIKV strain resulted in embryonic death whereas it caused microcephaly with Asian ZIKV strains. Together, our results demonstrate the high epidemic potential and pathogenicity of recent ZIKV strains from Africa. Importantly, they also imply that the African ZIKV lineage could more easily go unnoticed by public health surveillance systems than the Asian ZIKV lineage due to its propensity to cause fetal loss rather than birth defects.

Keywords: arbovirus; epidemiological dynamics; virus emergence; congenital Zika syndrome; Aedes aegypti; vertical transmission; spectrum of disease. 


\section{Introduction}

Zika virus (ZIKV) is a flavivirus mainly transmitted among humans through the bite of infected Aedes aegypti mosquitoes ${ }^{1,2}$. After its first isolation from a sentinel monkey in Uganda in 1947, ZIKV was shown to circulate in enzootic sylvatic cycles in Africa and continental Asia, but human infections were only sporadically reported for half a century $^{3-5}$. The first documented human epidemic of ZIKV occurred in 2007 on the Pacific island of Yap, Micronesia ${ }^{6}$. Subsequently, larger ZIKV outbreaks were recorded in French Polynesia and other South Pacific islands in 2013-2014 (refs. ${ }^{7,8}$ ). In May 2015, ZIKV was detected for the first time in Brazil from where it rapidly spread across the Americas and the Caribbean, causing an epidemic of unprecedented magnitude involving hundreds of thousands of human cases ${ }^{9}$. Whereas human ZIKV infections are usually asymptomatic or result in a self-limiting mild illness, ZIKV was associated for the first time with severe neurological complications such as Guillain-Barré syndrome (GBS) in adults, and congenital Zika syndrome (CZS), a spectrum of fetal abnormalities and developmental disorders including microcephaly, when mothers were infected during early pregnancy ${ }^{10,11}$. Within less than a decade, ZIKV went from a poorly known virus causing sporadic human infections in Africa and Asia to a nearly pandemic neurotropic virus with active circulation detected in a total of 89 countries and territories to date ${ }^{12}$. Phylogenetic analyses of ZIKV genetic diversity identified two major ZIKV lineages referred to as the African lineage and the Asian lineage, respectively ${ }^{13}$. Strikingly, all ZIKV strains responsible for human outbreaks to date belong to the Asian lineage ${ }^{2,14}$.

The explosiveness and magnitude of worldwide ZIKV emergence increased awareness and surveillance in regions with seemingly favorable conditions, such as Asia or Africa. Retrospective analyses of samples and surveillance programs in several Asian countries revealed that ZIKV had circulated at low but sustained levels for decades $^{15,16}$. Improved case recognition shed light on small outbreaks in Singapore ${ }^{17}$, Vietnam ${ }^{18}$ and India ${ }^{19}$ and led to the first reports of birth defects caused by indigenous ZIKV strains in South East Asia ${ }^{20-23}$. In Africa, where both ZIKV and Ae. aegypti mosquitoes are present, only one human outbreak was reported in the archipelago of Cape Verde between 2015 and 2017 (ref. ${ }^{24}$ ). Autochthonous ZIKV transmission was 
also detected in Angola during the same time period with four confirmed acute Zika cases and several suspected cases of microcephaly. Phylogenetic analyses revealed that the ZIKV strains detected in Cape Verde and Angola belonged to the Asian lineage and were probably independently imported from Brazil ${ }^{25,26}$. So far, the African ZIKV lineage has never been detected outside the African continent and never been associated with epidemic transmission, birth defects or neurological disorders ${ }^{27}$.

Surprisingly, a growing body of experimental evidence, both in vitro and in vivo, points towards a higher transmissibility and pathogenicity of the African ZIKV strains compared to their Asian counterparts. African ZIKV strains typically cause more productive and more lethal infections than Asian strains in cell culture ${ }^{28-34}$, they are more transmissible by mosquitoes ${ }^{35-39}$ and they are associated with more severe pathology in adult mice and mouse embryos ${ }^{34,40-48}$. A few studies, however, reported evidence supporting the opposite conclusion in non-human primates ${ }^{49-51}$, various cell types $^{52,53}$ and mosquitoes ${ }^{47}$. This discrepancy may reflect the lack of standard panels of ZIKV strains and/or the scarcity of recent African ZIKV strains available from public biobanks and laboratory collections ${ }^{54}$. Indeed, most of the available African ZIKV strains were isolated several decades ago and often underwent numerous passages in cell culture and/or suckling mouse brains ${ }^{55}$, questioning their biological relevance for comparative studies and experimental assessments of their epidemic potential.

To more rigorously assess the relative epidemic potential of the Asian and African ZIKV lineages, we compared their transmissibility by mosquitoes and pathogenicity in immunocompromised mice using a panel of seven recent, low-passage ZIKV strains representing the current viral genetic diversity. Using the newly generated empirical data and a previously described stochastic agent-based model ${ }^{56}$, we performed outbreak simulations in silico to quantify the epidemiological dynamics of each ZIKV strain. Finally, we used a mouse model of ZIKV-induced microcephaly to evaluate the ability of the ZIKV strains to disrupt embryonic development in utero. 


\section{Results}

To perform a comprehensive phenotypic characterization in both mosquito and mouse models, we assembled a set of seven recently isolated, low-passage ZIKV strains based on their broad phylogenetic coverage, worldwide geographical distribution and minimal passage history. Our ZIKV panel included two recent strains from the African lineage (Senegal_2011; Senegal_2015), three non-epidemic strains from the Asian lineage (Philippines_2012, Cambodia_2010, Thailand_2014) and two epidemic strains from the Asian lineage (F_Polynesia_2013, Puerto_Rico_2015) (Table S1; Fig. 1).

\section{African ZIKV strains are more transmissible by mosquitoes than Asian strains}

To evaluate ZIKV transmissibility by mosquitoes, we used a wild-type laboratory colony of Ae. aegypti initiated from a natural population in Barranquilla, Colombia in 2017. Female mosquitoes of the $4^{\text {th }}$ or $5^{\text {th }}$ laboratory generation were challenged by oral exposure to standardized infectious doses of ZIKV in artificial infectious blood meals. Upon exposure, blood-engorged mosquitoes were collected 7, 10, 14, and 17 days post blood meal and processed to detect ZIKV RNA in their body and infectious ZIKV in their saliva.

We first examined the mosquito infection rate (proportion of blood-fed mosquitoes with body infection) and transmission efficiency (proportion of blood-fed mosquitoes with infectious saliva) after oral exposure to a relatively high dose of the five Asian ZIKV strains and of the Senegal_2015 strain. Experimental variation in infectious dose was minimal between the ZIKV strains, ranging from 5.6 to $5.8 \log _{10}$ focus-forming units (FFU) per $\mathrm{ml}$ of artificial blood meal. Mosquito infection rates were consistently high, ranging from $82 \%$ to $100 \%$ across ZIKV strains and time points (Fig. 2A). They did not differ statistically at any of the time points with the exception of day 7 (logistic regression: $p=0.0155)$. In contrast, the transmission efficiency of the Senegal_2015 strain was significantly higher at all time points (logistic regression: $p=0.0224$ on day 7 and $p<0.0001$ at later time points), reaching $83 \%$ of infectious mosquitoes at the end of the time course (Fig. 2B). Infectious viral particles were detected in mosquito saliva as early as 7 days post blood meal for the Senegal_2015 strain and only after 10 days for the Cambodia_2010 strain, 14 days for the Philippines_2012, F_Polynesia_2013 
and Puerto_Rico_2015 strains, and 17 days for the Thailand_2014 strain. Final transmission efficiency also differed between Asian strains, ranging from $10 \%$ for the Thailand_2014 strain to 50\% for the F_Polynesia_2013 strain on day 17 post blood meal.

Next, we tested whether the superior transmissibility of the Senegal_2015 strain was representative of the African ZIKV lineage or specific to this strain. We conducted a second experiment that included the two African ZIKV strains of the panel and the F_Polynesia_2013 strain, as a representative Asian strain. To avoid saturation and increase our ability to detect differences in infection rate, we used a lower infectious dose (4.7-4.8 $\log _{10} \mathrm{FFU} / \mathrm{ml}$ ) than in the first experiment. The mosquito infection rate remained relatively high $(68 \%-93 \%)$ for the two African strains, whereas it was significantly lower at all time points for the F_Polynesia_2013 strain (logistic regression: $p=0.0384$ on day 17 and $p<0.0001$ at earlier time points), increasing from $24 \%$ on day 7 to $59 \%$ on day 17 (Fig. 2C). The difference was more striking (logistic regression: $p=0.1086$ on day 7 and $p<0.0003$ at later time points) for the transmission efficiency (Fig. 2D). Between day 7 and day 17, transmission efficiency of the Senegal_2015 and Senegal_2011 strains increased from 0\% to 52\% and from 7\% to $70 \%$, respectively, whereas no infectious particles were detected in any of the saliva samples collected from mosquitoes infected with the F_Polynesia_2013 strain throughout the time course. These results indicate that ZIKV strains of the African lineage, in general, display a significantly higher transmission potential than ZIKV strains of the Asian lineage.

To translate the observed variation in transmissibility between ZIKV strains into differences in epidemic risk, we incorporated our empirical data into a stochastic agentbased model to perform outbreak simulations in silico. The model consists of a population of mosquitoes that interacts with a population of humans in a simulated environment, accounting for infection dynamics within each host. Mosquito-to-human ZIKV transmission events were governed by parameters derived from our ZIKV strainspecific experimental data (Fig. 2A and 2B) whereas human-to-mosquito transmission events depended on shared parameters among the ZIKV strains. The probability of human-to-mosquito transmission over time was estimated from an independent experiment in which batches of naïve mosquitoes were allowed to feed daily on ZIKV- 
infected mice (Cambodia_2010 strain) during the course of their viremic period (Fig. S1). Simulations were initiated with one infected human and were run 100 times for 400 days or until the allowed number of infected individuals was reached $(100,000$ humans or $1,000,000$ mosquitoes). The magnitude of the outbreak was defined according to the number of secondary human infections, ranging from a lack of outbreak (no secondary infection), to small-scale outbreaks ( $<100$ secondary infections) and to large-scale outbreaks (>100 secondary infections). All ZIKV strains were able to cause secondary human infections, however the risk and magnitude of the outbreak greatly varied among the strains (Fig. 3). The proportion of simulations resulting in at least one secondary human infection ranged from 8\% (Thailand_2014) to $60 \%$ (Senegal_2015). The proportion of small-scale outbreaks ranged from $4 \%$ (Senegal_2015) to 22\% (Philippines_2012) and the proportion of large-scale outbreak ranged from 0\% (Puerto-Rico_2015; Thailand_2014) to 56\% (Senegal_2015). We did not observe any association between the epidemic or non-epidemic status of the ZIKV strains and the probability and magnitude of outbreaks. Together, the simulation results indicate that the higher transmissibility of the African ZIKV strains in our laboratory experiments translates into a markedly higher probability and size of human outbreaks predicted by our epidemiological model.

\section{African ZIKV strains are more lethal than Asian strains in immunocompromised adult mice}

To assess the ability of ZIKV strains to cause more or less severe disease in a mammalian model, we intraperitoneally inoculated AG129 mice (6-12 weeks of age) with $10^{3}$ plaque-forming units (PFU) of ZIKV (8-10 mice per strain). Upon infection, mice were monitored daily for changes in viremia, body weight and clinical signs of disease. In addition, blood was collected from alternating groups of mice from day 1 to day 6 post infection. Viremia levels ranged from 4.71 to $9.15 \log _{10}$ viral RNA copies/ml of plasma and peaked on day 3 post infection except for the Philippines_2012 strain, for which viremia peaked on day 2 (Fig. S2). Across the viremic period, the average viremia level was $7.04 \log _{10}$ viral RNA copies/ml for the African ZIKV lineage and 6.60 $\log _{10}$ viral RNA copies/ml for the Asian ZIKV lineage. Accounting for differences between strains within each ZIKV lineage, the level of viremia was lower on day 2 post infection (nested ANOVA: $p<0.0001$ ) and higher at all subsequent time points ( $p=0.0486$ on day 5 and $p<0.0001$ at other time points) for the African lineage 
compared to the Asian lineage (Fig. S2). During the first 6 days of infection, the average body weight of mice infected with Asian ZIKV strains was $100.2 \%$ (range: $85.8 \%-109.0 \%$ ) of their initial weight, however it was $92.8 \%$ (range: $74.4 \%-104.7 \%$ ) for mice infected with African ZIKV strains (Fig. 4A). Accounting for differences between strains within each ZIKV lineage, the body weight was significantly lower on day 2 post infection (nested ANOVA: $p=0.0468$ ) and at all subsequent time points $(p<0.0006)$ for the African lineage relative to the Asian lineage. Following infection, mouse survival differed significantly between ZIKV strains (log-rank test: $p<0.0001$ ). All mice infected with the African ZIKV strains became morbid and reached humane endpoints on day 6 post infection (Fig. 4B). In contrast, mice infected with the Asian strains developed disease symptoms significantly later and only started to reach humane endpoints from day 9 post infection onwards. Their median time to death ranged from 10 to 10.5 days, with the exception of the Thailand_2014 strain for which $50 \%$ of the mice were still alive at the end of the follow-up period (Fig. 4B). These results show that both of our African ZIKV strains are more pathogenic overall than their Asian counterparts in immunocompromised adult mice.

AG129 mice are highly permissive to ZIKV $^{57}$ and viremia levels may thus easily saturate when they are inoculated with a high dose of virus. To avoid saturation and enhance our ability to detect differences in viral RNA levels in plasma and tissues, and to delay the onset of disease in mice infected with the African ZIKV strains, we performed another experiment with a 1,000-fold lower inoculum (1 PFU). This experiment included both African ZIKV strains and two Asian ZIKV strains recapitulating the variation seen in the previous experiment. The Thailand_2014 strain was chosen for its attenuated phenotype and the F_Polynesia_2013 represented the other Asian ZIKV strains. Surprisingly, using a 1,000-fold lower inoculum delayed the onset of disease by only one day for mice infected with the African ZIKV strains since all these mice had to be euthanized on day 7 post infection. This result clearly highlighted the higher pathogenicity of the African ZIKV lineage. To enable a proper comparison of viral RNA levels in mouse tissues between ZIKV strains, all the other mice were also euthanized on day 7 post infection to collect their organs. Lowering the inoculum delayed the peak of viremia in all ZIKV-infected mice, which occurred on day 5 post infection for the F_Polynesia_2013 strain and on day 4 for the other ZIKV strains (Fig. 4C). The level of plasma viremia was significantly higher for the African ZIKV 
strains than for the Asian ZIKV strains at all time points (nested ANOVA: $p<0.001$ ). Likewise, viral RNA levels measured in organs collected on day 7 post infection were consistently higher for the mice infected with the African ZIKV strains (Fig. 4D). African ZIKV strains resulted in significantly higher viral loads than Asian strains in the brain (one-way ANOVA: $p<0.0001$ ), spinal cord (Brown-Forsythe and Welch ANOVA: $p<0.0001$ ), testis (one-way ANOVA: $p<0.0001$ ), kidney (Kruskal-Wallis rank-sum test: $p<0.0001$ ), and heart (one-way ANOVA: $p<0.0001$ ). These results indicate that whereas ZIKV strains from both lineages can cause systemic infections with similar organ tropism in this mouse model, the African strains are more pathogenic and result in significantly higher morbidity and mortality.

\section{African ZIKV strain causes fetal death in immunocompetent mice}

To investigate differences in vertical transmission phenotypes between ZIKV strains, we used a recent model of ZIKV-induced microcephaly by intraplacental injection in mouse embryos ${ }^{58,59}$. We first performed intraplacental injections of the Senegal_2015, Thailand_2014 and F_Polynesia_2013 ZIKV strains into the labyrinth of SWISS mouse embryos at E10.5 and compared the infection outcomes at E14.5. We observed subcutaneous edema in E14.5 embryos 4 days after intraplacental ZIKV injection for all strains (Fig. S3). Subcutaneous edema was significantly more frequent $(p<0.0012)$ in embryos infected with the Senegal_2015 strain $(91 \% ; n=11)$ than in embryos infected with the Thailand_2014 strain (30\%; n=16), the F_Polynesia_2013 strain (6\%; $n=16)$, or mock-injected embryos $(0 \% ; n=10)$. We next compared the extent of ZIKV systemic infection in E14.5 embryos following intraplacental injection at E10.5. ZIKV immunolabeling showed a comparable distribution of all ZIKV strains in brain, lung, heart, liver, intestine, eye, spinal cord and atriums of infected embryos (Fig. 5A; Fig. S4). The overall immunofluorescence staining was stronger for the Senegal_2015 and Thailand_2014 strains relative to the F_Polynesia_2013 strain. We confirmed these observations quantitatively by measuring viral RNA levels in the brain, lung, heart, liver and intestine. In all organs, viral loads differed significantly between ZIKV strains (oneway ANOVA: $p<0.0277$ ), with the F_Polynesia_2013 strain consistently resulting in significantly lower viral loads than the Senegal_2015 strain, as well as the Thailand_2014 strain in most organs (Fig. 5B). 
To measure the impact of different ZIKV strains on embryonic brain development, we performed intraplacental injections at E10.5 and examined embryos at E18.5. Injection of the Senegal_2015 strain caused massive resorption resulting in the death of all infected embryos harvested at E18.5 (Fig. 6A). In contrast, infection with the Asian ZIKV strains (F_Polynesia_2013 and Thailand_2014) was not lethal to E18.5 embryos but resulted in a significant reduction (Brown-Forsythe and Welch ANOVA: $p<0.05$ ) in head weight (Fig. 6C), cortical thickness (Fig. 6B and 6E) and number of cortical cells (Fig. 6F) compared to the mock-injected embryos. In addition, we detected a significant reduction in brain weight (Fig. 6D) and ventriculomegaly (Fig. 6B and 6G) with the Thailand_2014 strain but not the F_Polynesia_2013 strain. Together, these results show that ZIKV strains with higher levels of infection at E14.5 are also associated with more severe phenotypes at E18.5. The Senegal_2015 strain caused embryonic death before E18.5 and the Thailand_2014 strain resulted in more pronounced microcephaly and ventriculomegaly than the F_Polynesia_2013 strain.

\section{Discussion}

By comparing seven recent, low-passage ZIKV strains representing the breadth of viral genetic diversity worldwide, this study provides clear experimental evidence that recent African strains are more transmissible and potentially more pathogenic than Asian strains. In our experiments, ZIKV strains of the African lineage were more infectious to and were transmitted faster by wild-type Ae. aegypti mosquitoes from Colombia, translating into a higher epidemic potential in outbreak simulations. In addition, ZIKV strains of the African lineage were more pathogenic to immunocompromised adult mice and caused massive resorption and embryonic death in immunocompetent mouse embryos infected in utero by intraplacental injection.

We used immunocompromised AG129 mice (deficient in interferon $\alpha / \beta$ and $Y$ receptors) as a convenient proxy to assess pathogenesis in our panel of ZIKV strains. These mice are very susceptible to ZIKV infection ${ }^{57}$, making them highly suitable to monitor viral kinetics and disease manifestations. ZIKV strains of the African lineage caused significantly more morbidity and mortality than did their Asian counterparts, despite a similar tissue tropism. Of note, the level of pathogenicity observed in the 
immunocompromised adult mice model was not associated with the epidemic or nonepidemic status of the Asian ZIKV strains.

Our findings from the mosquito infection experiments and the immunocompromised mouse model support the hypothesis that worldwide ZIKV emergence in the last 15 years was not driven by adaptive virus evolution ${ }^{2}$. We found no evidence for enhanced transmission by the primary epidemic vector, $A e$. aegypti, or increased level and/or duration of viremia in the vertebrate host between epidemic Asian ZIKV strains (F_Polynesia_2013 and Puerto_Rico_2015) and non-epidemic Asian ZIKV strains (Cambodia_2010, Philippines_2012 and Thailand_2014). The Asian ZIKV strain that gave rise to the widespread epidemics in the Pacific and the Americas was probably not selected for its superior ability to infect mosquitoes and/or humans. Instead, it seems more likely that it was stochastically introduced through increased air travel and human mobility in regions with favorable epidemic conditions such as high densities of competent vectors and immunologically naïve human populations ${ }^{60}$.

The lack of human outbreaks associated with the African lineage of ZIKV until now ${ }^{27}$ is paradoxical because a large majority of experimental studies have found a higher transmissibility and pathogenicity of the African ZIKV strains relative to their Asian counterparts $^{28-34}$. We hypothesized that this discrepancy could have reflected the lack of recent, low-passage African strains available for experimental studies. Our panel included two ZIKV strains isolated in Senegal in 2011 and 2015, which are several decades more recent than most of the African ZIKV strains used in earlier studies. Our study unequivocally confirms that the African lineage of ZIKV is associated with higher transmissibility and pathogenicity. The reasons why African ZIKV strains have so far not been responsible for human outbreaks (or remained unnoticed) are unknown, but may include the lack of awareness prior to the worldwide emergence, the paucity of surveillance programs in resource-poor countries, protective effects of herd immunity or cross reactive antibodies, and/or the lower vectorial capacity of African mosquito populations.

Most ZIKV infections in humans are asymptomatic and the majority of symptomatic infections cause a non-specific acute febrile illness that can easily be mistaken for other common viral infections ${ }^{61}$. In the absence of severe infection outcomes such as 
GBS or CZS, low-level ZIKV circulation or even small-scale outbreaks could have gone unnoticed, especially before the emergence in the Pacific and Americas when ZIKV was still largely unknown. The worldwide emergence of ZIKV raised international awareness, resulting in improved surveillance including the implementation of epidemiological studies in regions where the virus was known to be present prior to the pandemic. Seroprevalence studies recently conducted across Africa generally found a low level $(<6.2 \%)$ of specific anti-ZIKV antibodies ${ }^{62-66}$. These studies are consistent with low-level ZIKV circulation in human populations of Africa and rule out the hypothesis that a high level of herd immunity is the main factor preventing ZIKV outbreaks in Africa. The alternative hypothesis that sustained circulation of closely related flaviviruses such as dengue virus (DENV) confers cross protection against ZIKV is also unlikely because high DENV seroprevalence has not prevented the emergence of ZIKV in South America, the Caribbean and the Pacific ${ }^{67}$.

A possible explanation for the lack of ZIKV outbreaks in Africa despite the high epidemic potential of African ZIKV strains is a reduced vectorial capacity of African $A e$. aegypti populations. ZIKV has been isolated from multiple mosquito species, but $A e$. aegypti is considered the main vector of transmission between humans in the urban cycle $^{1,2}$. In the absence of an efficient urban vector, human ZIKV infections in Africa would be limited to spillover transmission events from the sylvatic cycle via bridge vectors, which could explain the low level of ZIKV circulation ${ }^{66,68}$. Large-scale human outbreaks of dengue, yellow fever and chikungunya presumably mediated by $A e$. aegypti in Africa ${ }^{69,70}$ provide evidence that the density and human biting rate of African Ae. aegypti populations are sufficient to sustain urban transmission cycles. However, we recently discovered that African Ae. aegypti populations are significantly less susceptible to ZIKV infection than non-African populations using essentially the same panel of ZIKV strains as in the present study ${ }^{71}$. Although the Senegal_2015 strain was more infectious to mosquitoes relative to the other ZIKV strains, African Ae. aegypti populations were overall less susceptible than non-African Ae. aegypti populations regardless of the ZIKV strain ${ }^{71}$. This difference mirrors the existence of the two subspecies, Ae. aegypti aegypti and Ae. aegypti formosus, which were recognized by early taxonomists and later confirmed by modern population genetics ${ }^{72}$. Lower ZIKV susceptibility of the African subspecies Ae. aegypti formosus could have limited human ZIKV outbreaks in Africa in spite of the higher transmissibility of African ZIKV strains. 
This hypothesis is consistent with earlier reports of epidemic ZIKV transmission in Angola where the mosquito population consists of a genetic mixture of Ae. aegypti aegypti and Ae. aegypti formosus ${ }^{73}$.

An important implication of our study is that the African lineage of ZIKV should be considered a major threat to public health. Although African ZIKV strains have so far never been associated with human ZIKV outbreaks, our results with wild-type $A e$. aegypti from Colombia indicate that they may have greater epidemic potential than Asian strains if exported to regions where epidemic ZIKV transmission is realistic. We also point out that the African ZIKV strains may be associated with distinct clinical features allowing them to more easily escape surveillance systems. Our observations in an immunocompetent mouse model indicate that infection in utero by African ZIKV led to fetal death, rather than birth defects. Although this finding remains to be confirmed in humans, it is consistent with the lack of reported CZS in Africa. The only confirmed cases of birth defects in Africa were caused by ZIKV strains from the Asian lineage ${ }^{74}$. A few suspected cases were reported in Guinea-Bissau in 2016 where the African ZIKV lineage had been detected but these have never been confirmed ${ }^{75}$. On the other hand, CZS is a rare symptom and its absence may also simply reflect the lack of large-scale epidemics that would allow its detection.

It remains to be elucidated whether fetal harm has always been a possible consequence of ZIKV infection during pregnancy or whether ZIKV has recently acquired mutations conferring the ability to cause fetal harm. The recent detection of three CZS cases in Thailand and Vietnam suggest that both non-epidemic and epidemic Asian ZIKV strains are neurotropic and able to be vertically transmitted during pregnancy ${ }^{21-23}$. Our results, supported by recent studies performed in mouse models of vertical ZIKV transmission ${ }^{45,76}$, indicate that African ZIKV strains also possess the ability to cross the placenta and cause adverse perinatal outcomes. This reinforces the hypothesis that neurotropism and vertical transmission are not novel features of a recently emerged ZIKV variant, but rather an ancestral feature of ZIKV. In our intraplacental ZIKV challenge model, the non-epidemic Thailand_2014 strain was associated with more adverse outcomes than the epidemic F_Polynesia_2013 strain, whereas the Senegal_2015 strain led to fetal loss. Thus, ZIKV could have evolved 
bioRxiv preprint doi: https://doi.org/10.1101/2020.09.01.277673; this version posted September 1,2020 . The copyright holder for this preprint (which was not certified by peer review) is the author/funder, who has granted bioRxiv a license to display the preprint in perpetuity. It is made available under aCC-BY-NC-ND 4.0 International license.

towards attenuation by causing birth defects rather than fetal loss, supporting the counter-intuitive idea that attenuation was key to the recognition of ZIKV pathogenicity.

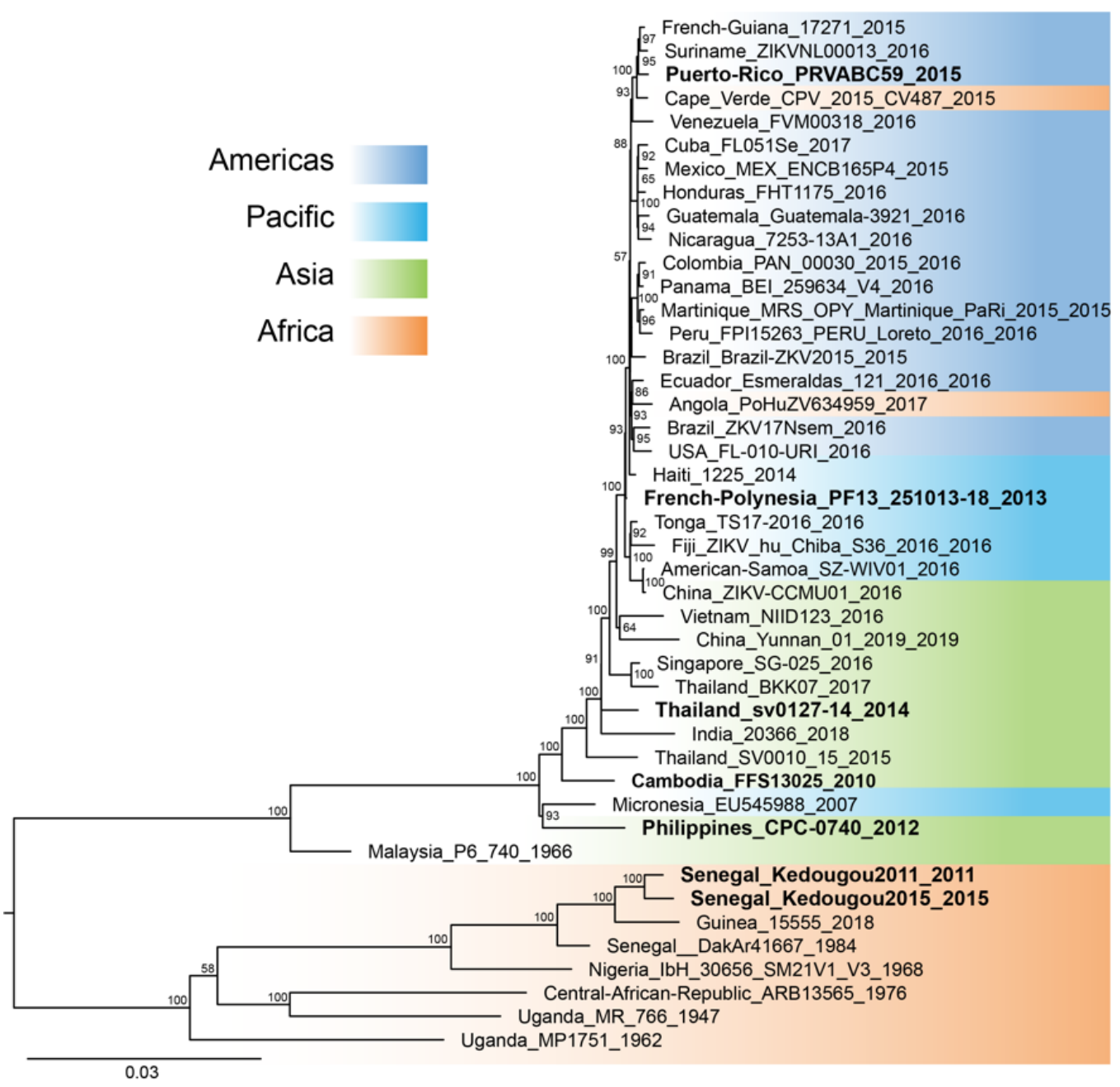

Fig. 1. Phylogenetic position of ZIKV strains used in this study. The phylogenetic tree shows the seven ZIKV strains of the panel (in bold) among a backdrop of ZIKV strains spanning the current viral genetic diversity. The colored background represents the geographic origin of ZIKV strains. The consensus tree was generated from 1,000 ultrafast bootstrap replicate maximum likelihood trees, using a GTR+F+G4 nucleotide substitution model of the full ZIKV open reading frame. The tree is midpoint rooted and the root position is verified by the Spondweni virus outgroup on amino-acid and codonbased trees. Support values next to the nodes indicate ultrafast bootstrap proportions (\%) and the scale bar represents the number of nucleotide substitutions/site. 
A

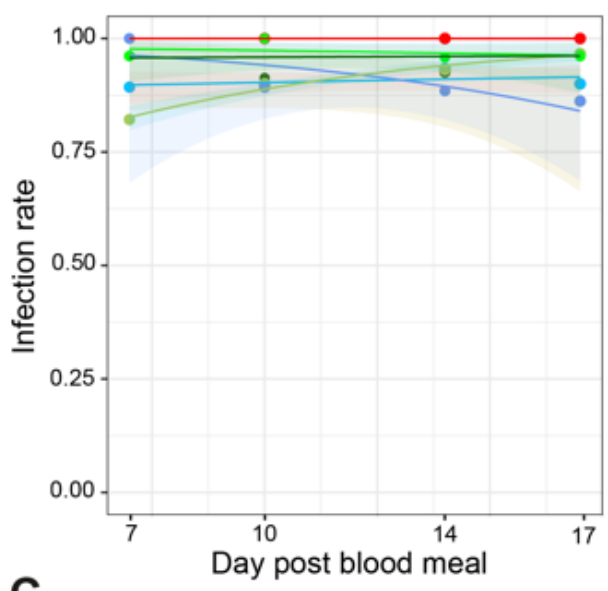

C

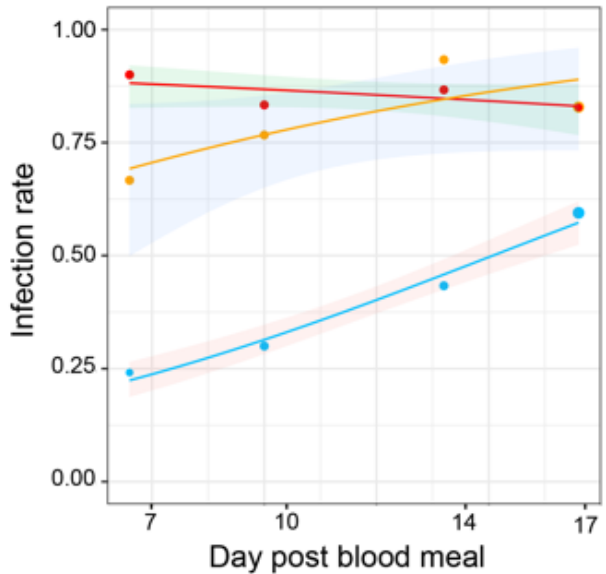

B

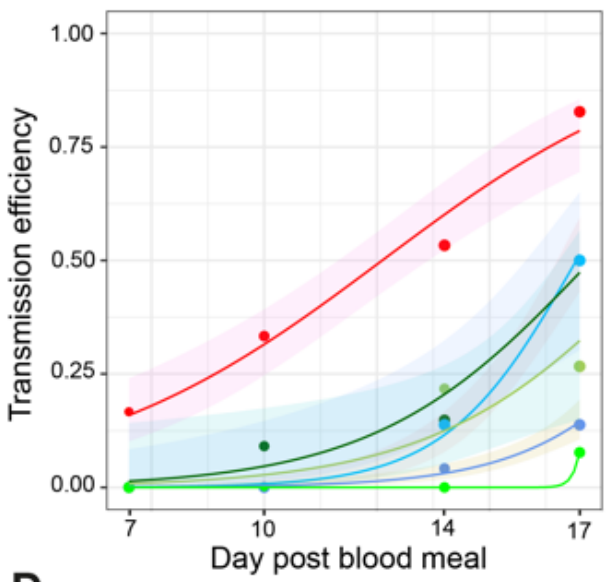

D

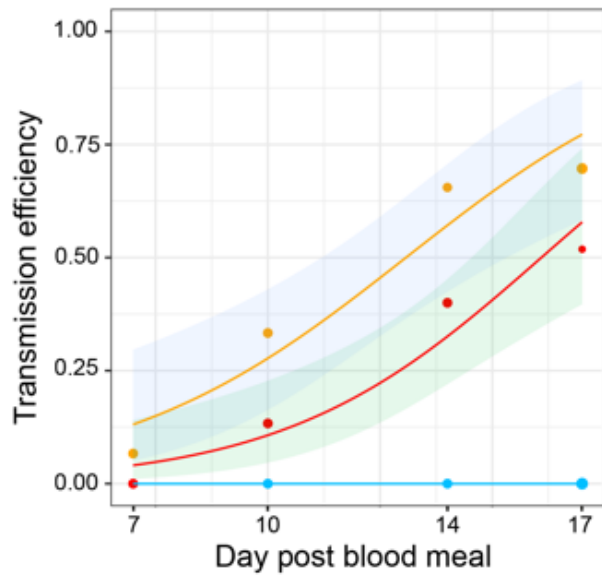

Asian lineage

F_Polynesia_2013

- Puerto_Rico_2015

Thailand_2014

O Philippines_2012

○ Cambodia_2010

African lineage

- Senegal_2015

Asian lineage

○ F_Polynesia_2013

African lineage

- Senegal 2015

○ Senegal_2011

Fig. 2. Mosquito infection rate and transmission efficiency of African and Asian ZIKV strains. Wild-type Ae. aegypti mosquitoes from Colombia were orally exposed to ZIKV and collected on day 7, 10,14 and 17 post infectious blood meal to analyze their carcasses and saliva samples collected in vitro. Infection rates and transmission efficiencies over time are shown for each ZIKV strain tested after oral exposure to a high dose (5.6-5.8 $\left.\log _{10} \mathrm{FFU} / \mathrm{ml}\right)$ (A, B) or a low dose (4.7-4.8 $\left.\log _{10} \mathrm{FFU} / \mathrm{ml}\right)$ (C, D) of virus. Infection rate is the proportion of ZIKV-positive carcasses among all blood-fed mosquitoes. Transmission efficiency is the proportion of blood-fed mosquitoes with infectious saliva. The line represents the logistic regression results and the shaded area is the $95 \%$ confidence interval of the logistic fit. The size of data points is proportional to the sample size (18-30 mosquitoes per group in the high-dose experiment and 29-37 mosquitoes per group in the low-dose experiment). 


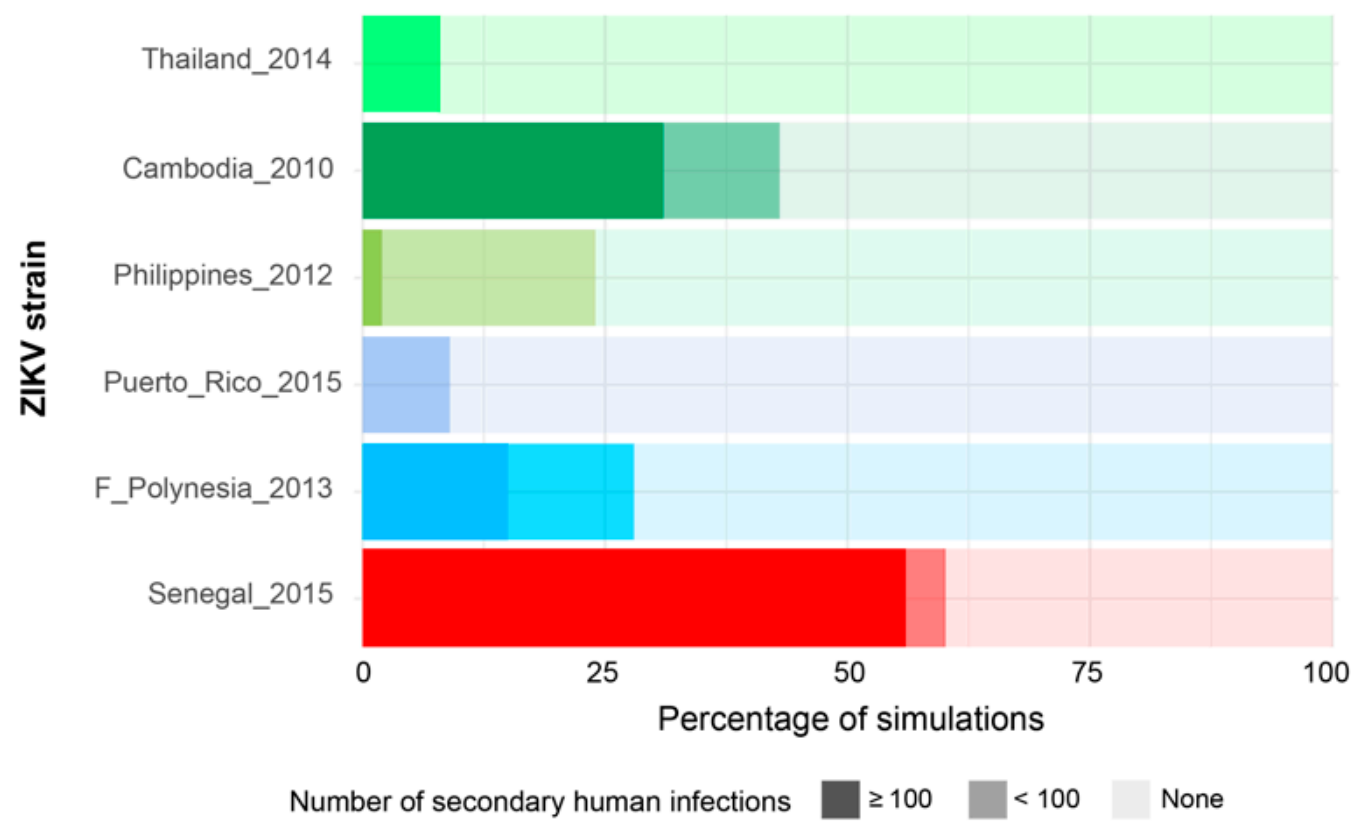

Fig. 3. Simulated effect of empirical variation in ZIKV transmissibility on the risk and magnitude of human outbreaks. A stochastic agent-based model was run 100 times based on the experimentally determined kinetics of mosquito infection and transmission of six ZIKV strains. Other parameters of the model, such as the mosquito biting rate and infection dynamics within the human host, were shared between viruses. The figure shows the proportion of simulated outbreaks that resulted in $\geq 100$, $<100$ and no secondary human infections. 
A

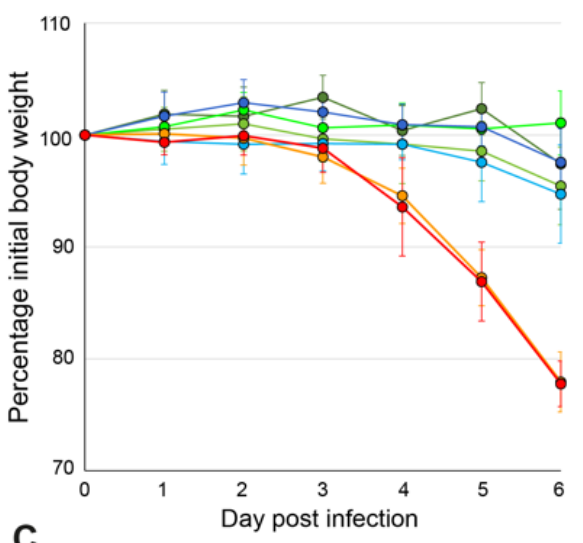

C

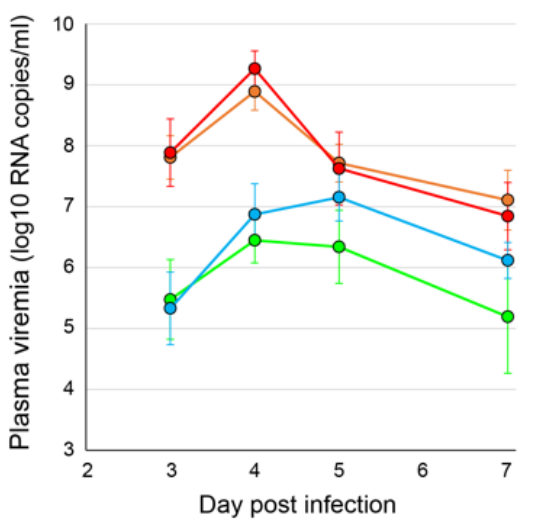

B

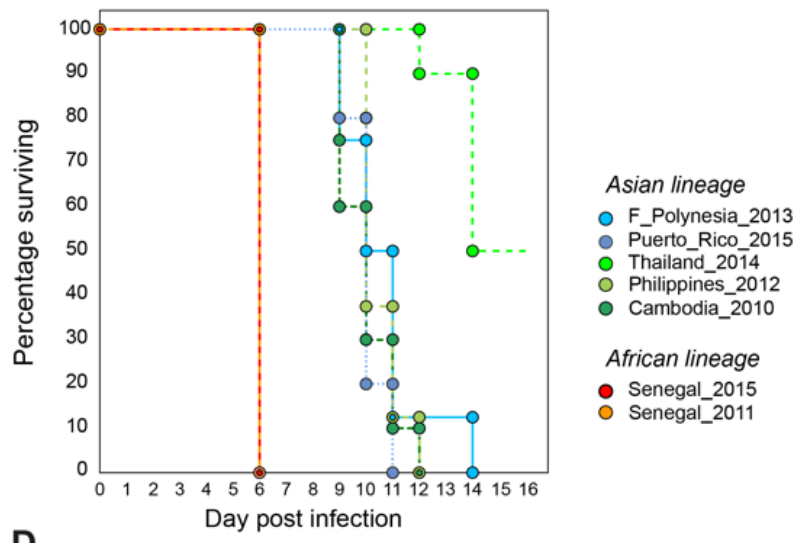

D

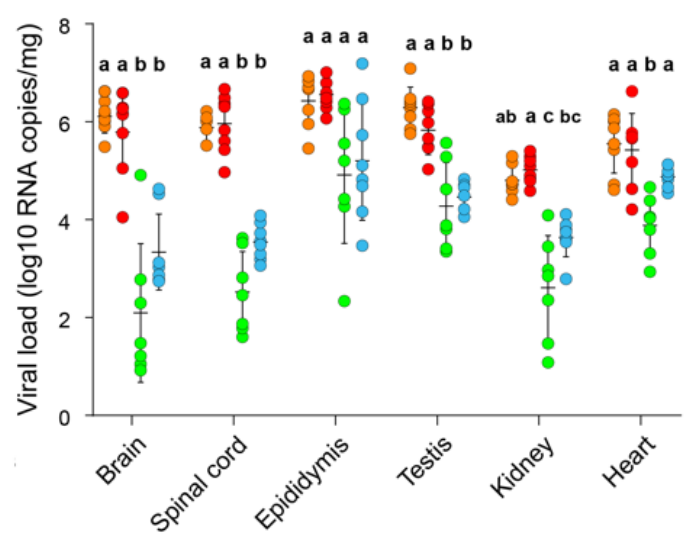

Fig. 4. Pathogenicity of African and Asian ZIKV strains in immunocompromised adult mice. In a first experiment (A-B), male AG129 mice were inoculated with $10^{3}$ PFU of ZIKV ( $n=8-10$ mice per strain). (A) Mouse weight over time is shown as the percentage of body weight prior to infection (mean \pm standard error). (B) Mouse survival over time is shown as the percentage of mice alive. Mice were euthanized when reaching humane endpoints (weight loss $>20 \%$ or/and severe symptom onset). In a second experiment (C-D), male AG129 mice were inoculated with 1 PFU of ZIKV ( $n=8$ mice per strain). (C) Time course of mouse viremia is shown in $\log _{10}$-transformed viral genome copies per $\mathrm{ml}$ of plasma (mean \pm standard error). Three extreme outliers were excluded for the Senegal_2015 strain. (D) Viral loads in organs collected on day 7 post infection are shown in $\log _{10}$-transformed viral genome copies per $\mathrm{mg}$ of tissue. Data are presented as mean \pm standard deviation. Statistical significance of differences was determined by one-way ANOVA followed by Tukey's post-hoc test for brain, heart and testis, by Brown-Forsythe and Welch ANOVA followed by GamesHowell's post-hoc test for epididymis and spinal cord, and by Kruskal-Wallis rank sum test followed by Dunn's post-hoc test for kidney. ZIKV strains not sharing a letter above the graph are statistically significantly different $(p<0.05)$. 
A

DAPI ACC3 ZIKV

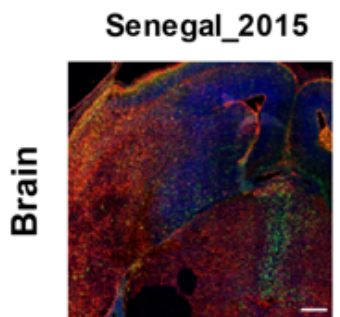

Thailand_2014

F_Polynesia_2013

Mock
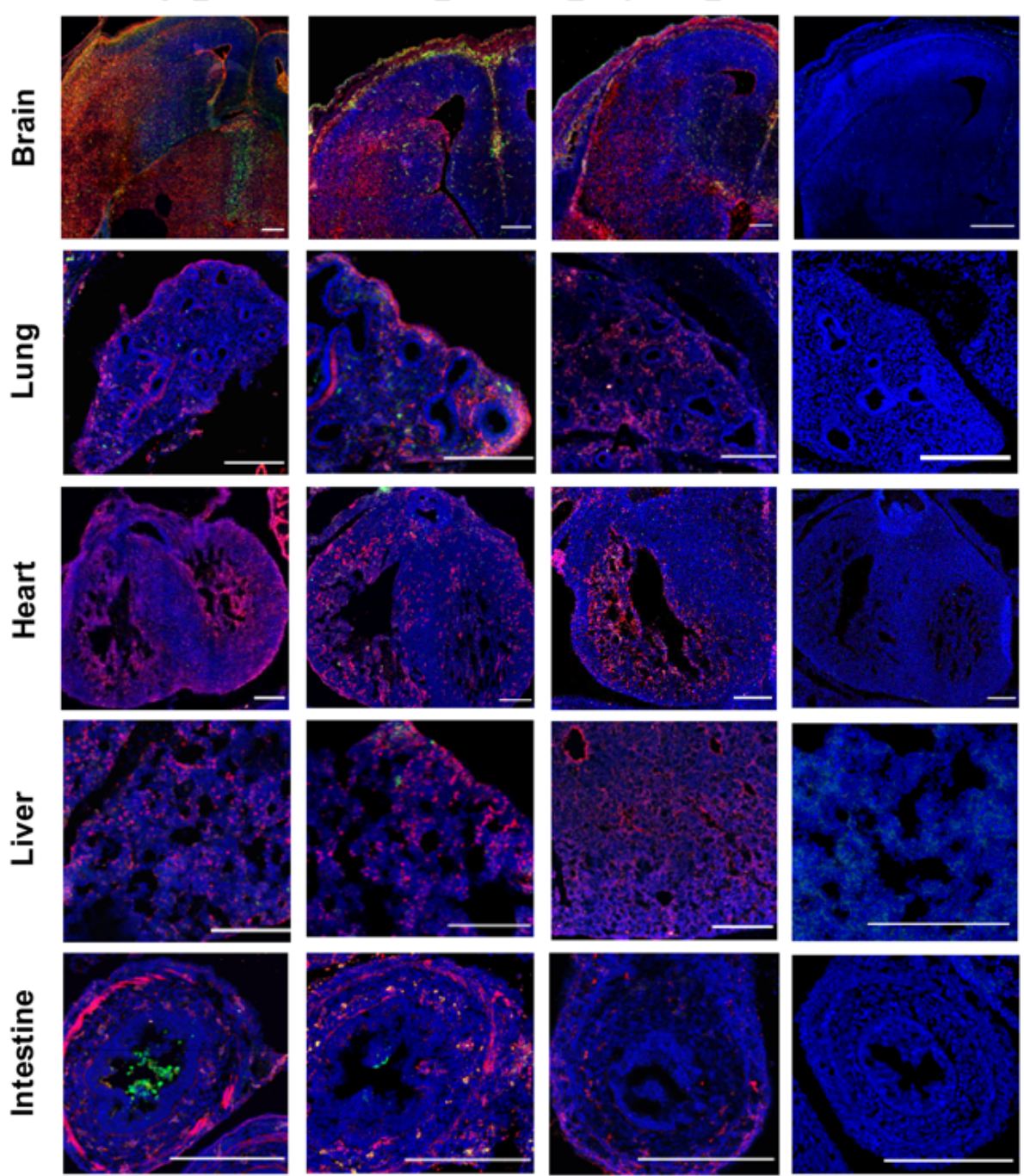

B

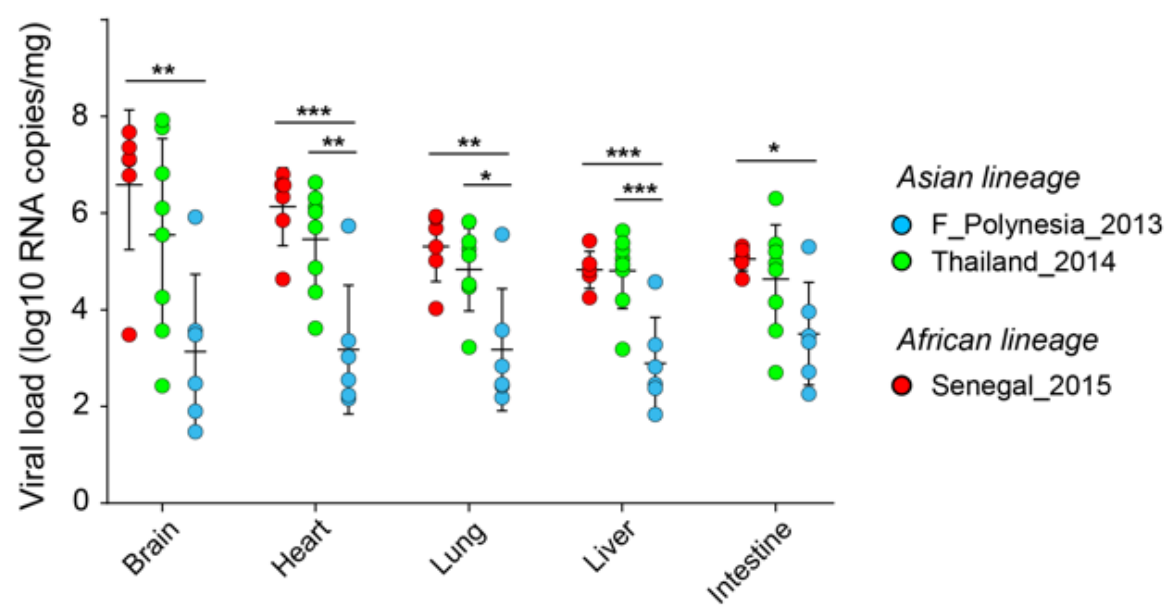

Fig. 5. Organ tropism and viral load of African and Asian strains of ZIKV in vertically infected mouse embryos. Immunocompetent mouse embryos were infected at E10.5 by intraplacental injection of 500-1,000 PFU of ZIKV and analyzed at E14.5 by microdissection. (A) Immunolabeling of embryonic brain, lung, heart, liver 
and intestine sections representative of each ZIKV strain tested ( $n=3$ embryos per strain). Blue, green and red colors indicate DAPI, anti-cleaved caspase 3 (ACC3) and ZIKV stainings, respectively. The scale bars represent $200 \mu \mathrm{m}$. (B) Viral load of embryonic brain, lung, heart, liver and intestine are shown for each ZIKV strain in viral genome copies per organ. Data are presented as mean \pm standard deviation. Statistical significance of the differences was determined by one-way ANOVA followed by Tukey's post-hoc test and is only shown when significant $\left({ }^{* *} p<0.001\right.$; ${ }^{* *} p<0.01$; $\left.{ }^{*} p<0.05\right)$.

A Mock Senegal_2015
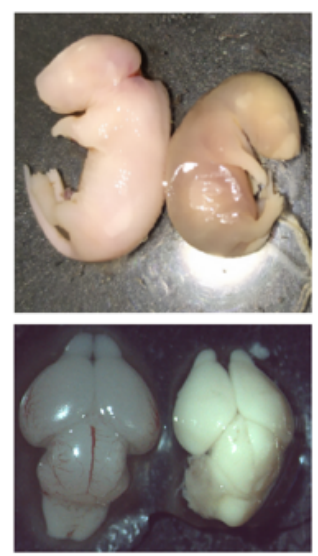

B
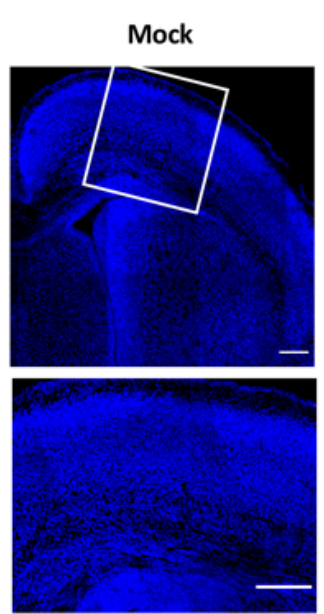

E

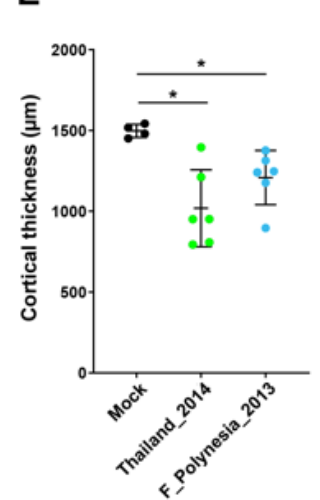

DAPI ACC3 ZIKV

Thailand_2014
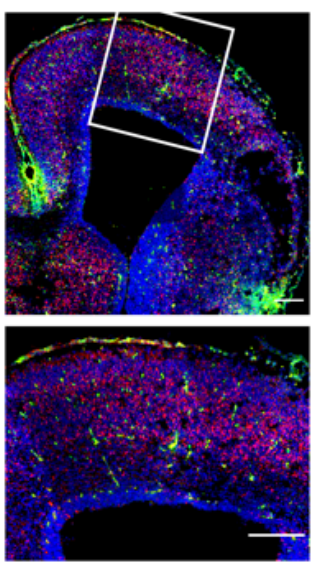

F

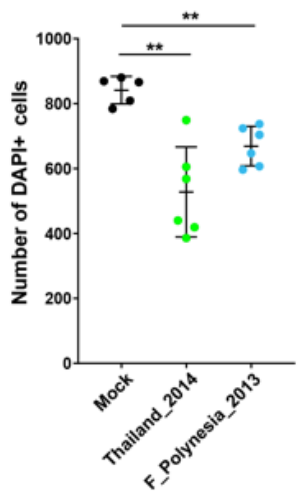

F_Polynesia_2013
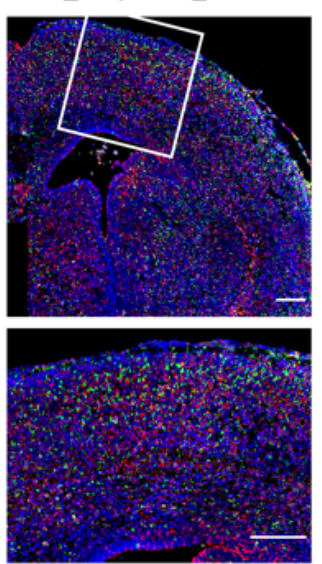

G

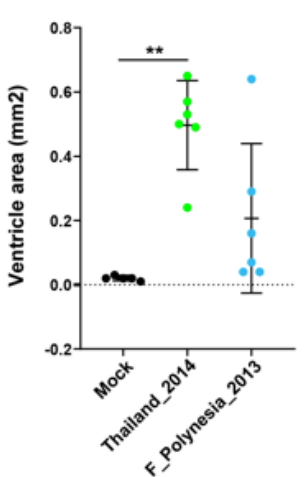

Fig. 6. Brain phenotypes of mouse embryos vertically infected with African and Asian strains of ZIKV. Immunocompetent mouse embryos were infected at E10.5 by intraplacental injection of 500-1,000 PFU of ZIKV and analyzed at E18.5 by microdissection. (A) Representative view of E18.5 embryos (top) and dorsal view of E18.5 embryonic brains (bottom) after mock injection (left; $n=10$ ) or infection by the 
Senegal_2015 ZIKV strain (right; $n=7)$. (B-G) Analyses of in utero brain development of E18.5 mouse embryos after mock injection $(n=7)$ or infection by the Thailand_2014 ( $n=8)$ or F_Polynesia_2013 ( $n=6)$ ZIKV strains. (B) Immunolabeling of embryonic brain sections representative of each ZIKV strain tested (top: full view; bottom: enlarged area within white frame). Blue, green and red colors indicate DAPI, anti-cleaved caspase 3 (ACC3) and ZIKV stainings, respectively. The scale bars represent $200 \mu \mathrm{m}$. (C, D) Embryonic heads and brains were examined morphologically by measuring (C) head weight and (D) brain weight normalized to head weight. (E, F) Microcephalic phenotypes were assessed by measuring (E) cortical thickness and (F) number of DAPI-positive cells. (G) Ventriculomegaly was estimated by measuring the ventricle area. In (C-G) data are presented as mean \pm standard deviation. Statistical significance of differences was determined by Brown-Forsythe and Welch ANOVA followed by Tamhane's T2 multiple comparison test. Only statistically significant differences are shown $\left({ }^{* * *} p<0.001 ;{ }^{* *} p<0.01 ;{ }^{*} p<0.05\right)$. 


\section{Methods}

\section{Ethics and regulatory information}

Human samples. This study used fresh human blood to prepare mosquito artificial infectious blood meals. For that purpose, healthy blood donor recruitment was organized by the local investigator assessment using medical history, laboratory results and clinical examinations. Biological samples were supplied through the participation of healthy adult volunteers at the ICAReB biobanking platform (BB-003300062/ICAReB platform/Institut Pasteur, Paris/BBMRI AO203/[BIORESOURCE]) of the Institut Pasteur in the CoSImmGen and Diagmicoll protocols, which had been approved by the French Ethical Committee Ile-de-France I. The Diagmicoll protocol was declared to the French Research Ministry under reference 343 DC 2008-68 COL 1. All human subjects provided written informed consent.

Animal experiments. The mouse experiments conducted at Institut Pasteur were approved by the Institut Pasteur Animal Ethics Committee (project number dap170045) and authorized by the French Ministry of Research (authorization number 12861). The Institut Pasteur animal facility had received accreditation from the French Ministry of Agriculture to perform experiments on live animals in compliance with the French and European regulations on the care and protection of laboratory animals (authorization number 75-15-01). Mouse experiments conducted in Belgium strictly followed the Belgian guidelines for animal experimentation and the guidelines of the Federation of European Laboratory Animal Science Associations. Mouse experiments were performed with the approval of the Ethical Committees of the Animal Research Center of KU Leuven (authorization number P019-2016) and of the University of Liège (authorization number 16-1837), in accordance with the guidelines of the Belgian Ministry of Agriculture, and in agreement with the European Community Laboratory Animal Care and Use Regulations (86/609/CEE, Journal Officiel des Communautés Européennes L358, 18 December 1986).

\section{ZIKV strains}

Seven low-passage ZIKV strains were chosen based on their geographical origin and year of isolation to best represent the current breadth of ZIKV genetic diversity (Table S1). ZIKV strains were obtained from the World Reference Center for Emerging 
Viruses and Arboviruses at the University of Texas Medical Branch (PRVABC59, FSS13025), the Armed Forces Research Institute of Medical Sciences (PHL/2012/CPC-0740, THA/2014/SV0127-14), the Institut Louis Malardé in French Polynesia (PF13/251013-18) and the Institut Pasteur in Dakar (Kedougou2011, Kedougou2015). High-titered stocks were prepared and their infectious titers were measured by focus-forming assay (FFA) as previously described ${ }^{77,78}$ or by plaque assay as previously described ${ }^{57}$. For FFA, a commercial mouse anti-flavivirus group antigen monoclonal antibody (MAB10216; Merck Millipore) diluted 1:1,000 in phosphate-buffered saline (PBS; Gibco Thermo Fisher Scientific) supplemented with $1 \%$ bovine serum albumin (BSA; Interchim) was used as the primary antibody. The secondary antibody was an Alexa Fluor 488-conjugated goat anti-mouse antibody (A11029; Life Technologies) diluted 1:500 in PBS supplemented with 1\% BSA.

\section{Genome sequencing of ZIKV strains}

The seven ZIKV strains of the panel were sequenced as previously described 25,77 . Briefly, RNA was extracted from virus stock using QIAamp Viral RNA Mini Kit (Qiagen) and treated with TURBO DNase (Ambion). The Senegal_2011 and Senegal_2015 strains also underwent depletion of host ribosomal RNA following a homemade protocol $^{25}$. cDNA was produced with random hexameric primers (Roche) using M-MLV (Invitrogen) or Superscript IV (Thermo Fisher Scientific) reverse transcriptase. After second-strand synthesis with Second Strand Synthesis Buffer (New England BioLabs), dsDNA was used for library preparation using Nextera XT DNA Kit (Illumina) or NEBNext Ultra II RNA Library Prep kit (New England Biolabs) according to the manufacturer's instructions. The final libraries were checked on a Bioanalyzer (Agilent) and combined with other libraries from unrelated projects to be sequenced on an Illumina NextSeq 500 instrument (150 cycles, paired ends). Raw sequencing datasets were deposited to the European Nucleotide Archive database under accession number PRJEB39677. The sequencing data were processed following a pipeline described elsewhere $^{79}$. Briefly, nucleotides with a quality score $<30$ were trimmed using Trimmomatic v0.36 (ref. ${ }^{80}$ ). Reads were filtered against the Aedes albopictus reference genome using Bowtie2 v2.3.4.3 (ref. ${ }^{81}$ ) and the remaining reads were subjected to de novo assembly with the Ray v2.3.1-mpi tool ${ }^{82}$ or metaSPAdes ${ }^{83}$. Scaffolds were subjected to a blastn search in the nucleotide NCBI database using BLAST v2.2.40 (ref. ${ }^{84}$ ). The closest hit was used to produce a chimeric genome 
sequence that served as a reference to re-map the filtered reads with Bowtie2 v2.3.4.3 and generate a consensus sequence.

\section{Phylogenetic analyses}

Genome sequences of ZIKV and Spondweni virus were retrieved from GenBank. The nucleotide sequences were aligned using MAFFT ${ }^{85}$. The phylogenetic analyses were performed based on nucleotide (open reading frame), amino-acid, and codon alignments using the maximum-likelihood method with substitution models (GTR+F+G4, FLU+G+R3, and SCHN05+FU+R4, respectively) selected with ModelFinder $^{86}$. Support for the tree was assessed with 1,000 ultrafast bootstrap replicates $^{87}$. The consensus trees were reconstructed with IQ-TREE v1.6.3 (ref. ${ }^{88}$ ) and visualized in FigTree v1.4.4 (ref. ${ }^{89}$ ). The phylogenetic tree root position was in agreement among the nucleotide-based tree without an outgroup (midpoint), aminoacid or codon tree with Spondweni virus as an outgroup, as well as with previously published ZIKV phylogenetic trees ${ }^{90}$. In addition to the seven genome sequences generated in this study, the alignment used to construct the tree included 37 sequences from GenBank (accession numbers: MK241416; MF574587; KX198135; KU647676; KY693679; KU497555; MF794971; MK829154; MH882540; KY014295; MH063262; KY631494; KY693677; MF434522; MF801378; KU758877; KU937936; KY693680; KU509998; KX806557; LC191864; KU963796; MF036115; LC219720; MN190155; KY241695; MH013290; MK238037; KX051562; EU545988; KX377336; MN025403; MF510857; KU963574; KF268948; MK105975; KY288905).

\section{Mosquitoes, mice and cell lines}

Mosquitoes. All mosquito experiments used the $4^{\text {th }}$ and $5^{\text {th }}$ generations of an $A e$. aegypti colony established from wild specimens caught in Barranquilla, Colombia, with the exception of the mouse-to-mosquito transmission experiment that used the $9^{\text {th }}$ generation of an Ae. aegypti colony from Saint François, Guadeloupe. Mosquitoes were reared under controlled insectary conditions as previously described ${ }^{77}$.

Mouse strains. In-house-bred, 6- to 12-week-old male AG129 mice (Marshall BioResources, Hull, UK), deficient in both interferon (IFN)- $\alpha / \beta$ and IFN- $\gamma$ receptors, were used for experimental ZIKV infections. In-house-bred, 10-week-old male and female 129S2/SvPas mice deficient for IFN- $\alpha / \beta$ receptors (Ifnar1 ${ }^{-/}$), were used for the mouse-to-mosquito ZIKV transmission assay. Time-mated, wild-type 
immunocompetent SWISS mice (Janvier Labs, Saint Berthevin, France) were used for ZIKV vertical transmission experiments.

Cell lines. The Aedes albopictus cell line C6/36 (ATCC CRL-1660) was used for amplification of all virus stocks and testing of mosquito saliva samples. The Cercopithecus aethiops cell line Vero (ATCC CCL-81) was used for titration of virus stocks by FFA. The C. aethiops cell line Vero E6 (ATCC CRL-1586) was used for titration of virus stocks by plaque assay. Cell lines were maintained as previously described ${ }^{57,78}$.

\section{Mosquito exposure to ZIKV via artificial blood meals}

Mosquitoes were orally challenged with ZIKV by membrane feeding as previously described ${ }^{77}$. Briefly, seven-day-old females deprived of sucrose solution for 24 hours were offered an artificial infectious blood meal for 15 min using a Hemotek membranefeeding apparatus (Hemotek Ltd.) with porcine intestine as the membrane. Blood meals consisted of a 2:1 mix of washed human erythrocytes and ZIKV suspension. Adenosine triphosphate (Merck) was added to the blood meal as a phagostimulant at a final concentration of $10 \mathrm{mM}$. Fully engorged females were sorted on wet ice, transferred into 1-pint cardboard containers and maintained under controlled conditions $\left(28^{\circ} \pm 1^{\circ} \mathrm{C}, 12 \mathrm{~h}: 12 \mathrm{~h}\right.$ light:dark cycle and $70 \%$ relative humidity) in a climatic chamber with permanent access to $10 \%$ sucrose solution. After $7,10,14$, and 17 days of incubation, mosquitoes were paralyzed with triethylamine to collect their saliva in vitro. The proboscis of each female was inserted into a $20-\mu l$ pipet tip containing $10 \mu \mathrm{l}$ of fetal bovine serum (FBS). After 30 min of salivation, the saliva-containing FBS was mixed with $30 \mu \mathrm{l}$ of Leibovitz's L-15 medium (Gibco Thermo Fisher Scientific), and stored at $-80^{\circ} \mathrm{C}$ for later testing. The saliva samples were subsequently thawed and inoculated onto C6/36 cells for ZIKV detection by FFA as described above without subsequent dilution. Mosquito bodies were homogenized individually in $300 \mu \mathrm{l}$ of squash buffer (Tris $10 \mathrm{mM}, \mathrm{NaCl} 50 \mathrm{mM}$, EDTA $1.27 \mathrm{mM}$ with a final pH adjusted to 8.2) supplemented with $1 \mu$ of proteinase K (Eurobio Scientific) for $55.5 \mu$ l of squash buffer. The body homogenates were clarified by centrifugation and $100 \mu$ of each supernatant were incubated for $5 \mathrm{~min}$ at $56^{\circ} \mathrm{C}$ followed by $10 \mathrm{~min}$ at $98^{\circ} \mathrm{C}$ to extract viral RNA. Detection of ZIKV RNA was performed using a two-step RT-PCR reaction to generate a 191-bp amplicon located in a conserved region of the ZIKV genome between the 3' end of the NS1 gene and the 5' end of the NS2A gene. Total RNA was 
reverse transcribed into cDNA using random hexameric primers and the M-MLV reverse transcriptase (Thermo Fisher Scientific) according to the following program: $10 \mathrm{~min}$ at $25^{\circ} \mathrm{C}, 50 \mathrm{~min}$ at $37^{\circ} \mathrm{C}$ and $15 \mathrm{~min}$ at $70^{\circ} \mathrm{C}$. The cDNA was subsequently amplified using DreamTaq DNA polymerase (Thermo Fisher Scientific). For this step, $20-\mu$ l reaction volumes contained $1 \times$ of reaction mix and $10 \mu \mathrm{M}$ of each primer (forward: 5'-GTATGGAATGGAGATAAGGCCCA-3'; reverse: ACCAGCACTGCCATTGATGTGC-3'). The thermocycling program consisted of 2 min at $95^{\circ} \mathrm{C}, 35$ cycles of $30 \mathrm{sec}$ at $95^{\circ} \mathrm{C}, 30 \mathrm{sec}$ at $60^{\circ} \mathrm{C}$, and $30 \mathrm{sec}$ at $72^{\circ} \mathrm{C}$ with a final extension step of $7 \mathrm{~min}$ at $72^{\circ} \mathrm{C}$. Amplicons were visualized by electrophoresis on a $2 \%$ agarose gel.

\section{Mouse-to-mosquito ZIKV transmission assay}

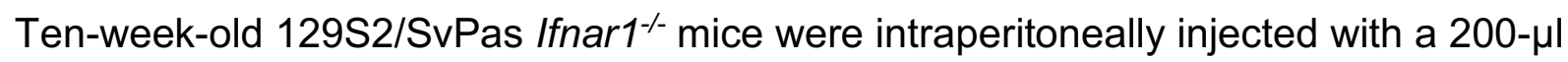
inoculum containing $10^{5}$ focus-forming units (FFU) of ZIKV (Cambodia_2010 strain). From day 1 to day 5 post inoculation, mice were anesthetized daily using $80 \mathrm{mg} / \mathrm{kg}$ of ketamine and $5 \mathrm{mg} / \mathrm{kg}$ of xylazine administered by the intraperitoneal route. Each anesthetized mouse was placed on the netting-covered top of a 1-pint cardboard box containing 25 two- to four-day-old $A e$. aegypti females previously deprived of sucrose solution for 24 hours. Mosquitoes were allowed to blood feed on the mouse for $15 \mathrm{~min}$. Fully engorged females were sorted on wet ice, transferred into fresh 1-pint cardboard containers and maintained under controlled conditions $\left(28^{\circ} \pm 1^{\circ} \mathrm{C}, 12 \mathrm{~h}: 12 \mathrm{~h}\right.$ light:dark light cycle and $70 \%$ relative humidity) in a climatic chamber with permanent access to $10 \%$ sucrose solution. After 14 days of incubation, saliva and bodies were collected and analyzed by FFA and RT-PCR as described above.

\section{ZIKV experimental infections of immunocompromised mice}

Survival. Mice were intraperitoneally injected with $10^{3}$ PFU of ZIKV $(2 \times 5$ mice per ZIKV strain). Upon infection, mice were observed daily for changes in body weight and clinical symptoms of virus-induced disease including dehydration, hunched back, and paralysis. Mice were euthanized when body weight loss was $>20 \%$ or when other humane endpoints were met according to the ethical guidelines. During days 1-8 of infection, mice were bled by submandibular puncture to monitor viremia kinetics. Plasma viremia was measured every other day from two alternating sub-groups of 5 mice each. Upon euthanasia, the brain, spinal cord, testes, and epididymes were 
collected and blood was collected by intracardiac puncture. After collection, tissues were immediately placed on dry ice and stored at $-80^{\circ} \mathrm{C}$ until further processing.

Tissue tropism. Mice were intraperitoneally injected with 1 PFU (8 mice per ZIKV strain). Upon infection, mice were observed daily for changes in body weight and clinical symptoms of virus-induced disease in case euthanasia would be required based on humane endpoints. Mice were bled by submandibular puncture on days 3 , 4, and 5 post infection. On day 7 post infection, all animals were sacrificed and blood was collected by intracardiac puncture. All animals were euthanized by intramuscular injection of Dolethal (Vétoquinol). The brain, spinal cord, testis, epididymis, heart, liver and kidney were collected after transcardial perfusion with PBS. After collection, tissues were immediately placed on dry ice and stored at $-80^{\circ} \mathrm{C}$ until further processing.

\section{ZIKV vertical transmission in immunocompetent mice}

Intraplacental injections. Timed-mated, wild-type pregnant SWISS dams were housed under standard conditions and allowed to acclimate for at least $24 \mathrm{~h}$ upon receipt with access to food and water ad libitum. The surgeries were performed at the same time of day, with noon of the day after mating set as embryonic (E) 0.5. Preoperative analgesia was administered subcutaneously with $0.1 \mathrm{mg} / \mathrm{kg}$ of buprenorphine (Temgesic) before induction of anesthesia with isoflurane (Abbot Laboratories Ltd.) in an oxygen carrier. A 1.0- to 1.5-cm incision was performed through the lower ventral peritoneum and the uterine horns were careful extracted onto warm humidified gauze pads. The intraplacental injections of embryos at E10.5 were performed as previously described ${ }^{58}$ with minor modifications. The fast green dye concentration was $0.05 \%$ and placenta was injected with either ZIKV or mock medium. The animals were randomly assigned to receive a 1.0- to 2.0- $\mu$ injection of mock medium or ZIKV stock containing $5 \times 10^{5} \mathrm{PFU} / \mathrm{ml}$.

Immunohistochemistry. After dissection, E18.5 mouse heads and E14.5 embryos were fixed in $4 \%$ paraformaldehyde in PBS for $24 \mathrm{~h}$ at $4{ }^{\circ} \mathrm{C}$. Brains were dissected in $0.1 \mathrm{M}$ PBS (pH 7.4). E18.5 brains and E14.5 embryos were cryoprotected (20\% sucrose in PBS) before being embedded in OCT (Richard-Allan Scientific Neg-50 Frozen Section Medium, Thermo Scientific) for cryosectioning 14- $\mu \mathrm{m}$ sections for brains and $20-\mu \mathrm{m}$ sections for embryos (Leica) onto slides (SuperFrost Plus, VWR International). Fluorescence immunohistochemistry was performed as previously described ${ }^{58}$. Briefly, a solution of antigen retrieval (Dako Target Retrieval Solution) was pre-heated at $95^{\circ} \mathrm{C}$ 
for 40 min and antigen retrieval of mouse brains and whole embryos were performed at $95^{\circ} \mathrm{C}$ for $5 \mathrm{~min}$ before incubation with primary antibodies. The primary antibodies were rabbit anti-cleaved caspase 3 (1:300, \#9661, Cell Signaling Technologies), mouse anti-flavivirus group antigen (1:800, MAB10216, Merck Millipore) and goat antiIba1 (1:300, ab5076, Abcam). The respective secondary antibodies were donkey antirabbit, anti-mouse and anti-goat antibodies conjugated with Alexa Fluor-488, Alexa Fluor-555, and Alexa Fluor-647 (A-21206, A-31570, A-21447, Life Technologies) and diluted 1:1,000. Nuclei were counterstained with DAPI (1:1,000, Sigma) and mounted in Dako Fluorescence Mounting Medium (Agilent).

Image acquisition and processing. Immunofluorescence images of embryonic brains (E18.5) and internal organs (E14.5) were acquired in magnified fields (20x and 25x) with either Nikon A1 or Zeiss LSM 880 AiryScan Elyra S.1 confocal microscopes and further processed with ImageJ 1.42q 276 (Wayne Rasband, National Institutes of Health), Fiji (v2.0.0-rc-54/1.51 h, https://imagej.net/Fiii) and Zen (Blue edition, Carl Zeiss Microscopy $\mathrm{GmbH}$ ) software.

\section{ZIKV RNA isolation and quantification in mouse samples}

Total RNA was isolated from microdissected embryonic mouse (E14.5) tissues using Trizol (Ambion, Life Technologies) according to the manufacturer's protocol. For adult mouse samples, sections of whole tissue were weighed and transferred to 2-ml Precellys tubes containing 2.8-mm zirconium oxide beads (Bertin Instruments). RLT lysis buffer (RNeasy Mini Kit, Qiagen) was added at a ratio of 19 times the weight of the tissue section. Tissue sections were homogenized in three cycles at $6,800 \mathrm{~Hz}$ with $30-\mathrm{sec}$ intervals using the Precellys24 homogenizer (Bertin Instruments). Homogenates were cleared by centrifugation ( $10 \mathrm{~min}, 14,000 \mathrm{rcf}, 4^{\circ} \mathrm{C}$ ) and total RNA was extracted from the supernatant using the RNeasy Mini Kit (Qiagen) according to the manufacturer's protocol. For plasma samples, viral RNA was extracted using the NucleoSpin RNA kit (Macherey-Nagel) following the manufacturer's instructions. Viral RNA was eluted in $50 \mu$ l of RNase-free water. Quantification of ZIKV genome copy numbers was performed by quantitative reverse transcription PCR (qRT-PCR) using the Applied Biosystems 7500 Fast Real-Time PCR System (Thermo Fisher Scientific). The Asian ZIKV strains were detected and quantified using a specific primer pair (forward: reverse: 
FAM/AGCCTACCT/ZEN/TGACAAGCAATCAGACACTCAA/3'IABkFQ

(Integrated DNA Technologies). The African ZIKV strains were detected and quantified using the same probe as for the Asian ZIKV strains but a different primer pair (forward: 5'GTCGCTGTCCAACACAAG-3', reverse: 5'-CACCAGTGTTCTCTTGCAGACAT-3') to accommodate several mismatches. Standard curves were generated based on 10-fold serial dilutions of gBlock synthetic oligonucleotides (Integrated DNA Technologies) whose sequences were specific to the Asian (5'GAGGCATCAATATCAGACATGGCTTCTGACAGCCGCTGCCCAACACAAGGTGAA GCCTACCTTGACAAGCAATCAGACACTCAATATGTCTGCAAAAGAACGTTAGTG GACAGAGGCTGGGGAAATGGATGTGGACT-3') and African

(5'GAGGCATCAATATCGGACATGGCTTCGGACAGTCGCTGTCCAACACAAGGTGA AGCCTACCTTGACAAGCAATCAGACACTCAATATGTCTGCAAGAGAACACTGGT GGATAGAGGTTGGGGAAATGGGTGTGGACT-3') ZIKV lineages. Ct values were converted into a relative number of ZIKV RNA copies/mg of tissue or $\mathrm{ml}$ of plasma using the formula $y=a^{*} \ln (x)+b$, where $a$ is the slope of the standard curve, $b$ is the $y$ intercept of the standard curve and $y$ is the Ct value for a specific sample.

\section{Statistics}

Statistical analyses were performed using JMP v10.0.2 (www.jmpdiscovery.com), GraphPad Prism v8.02 (www.graphpad.com) and the packages car, userfriendlyscience and DescTools of R v3.6.0 (www.r-project.org). Binary variables were analyzed by logistic regression followed likelihood-ratio $\chi^{2}$ tests. Continuous variables were analyzed by analysis of variance (ANOVA) when the underlying assumptions were satisfied. They were analyzed by Brown-Forsythe and Welch ANOVA when the homoscedasticity assumption was unmet and by Kruskal-Wallis rank sum test when both the normality and the homoscedasticity assumptions were unmet. Viremia levels were $\log _{10}$-transformed and compared using nested ANOVA with ZIKV strain nested within lineage. Survival curves were compared by log-rank test. Differences were considered statistically significant when $p<0.05$.

\section{Epidemiological modeling}

Model overview. To assess the epidemiological potential of ZIKV strains from empirically observed variation in mosquito infection dynamics, stochastic agent-based simulations were performed using the $\mathrm{R}$ package nosoi ${ }^{56}$. This modeling framework 
accounts for the influence of within-host infection dynamics on transmission probability during mosquito-human infectious contacts in a full epidemiological context ${ }^{91}$. Virus transmission was assumed to only occur either between an infected human and an uninfected mosquito, or between an infected mosquito and an uninfected human. Sexual and vertical transmission modes were considered epidemiologically insignificant ${ }^{92,93}$ and ignored in the model, and so were superinfections. The human and mosquito populations were considered homogeneous. Mosquito daily survival probability was set to 0.85 (ref. ${ }^{94}$ ). The daily number of mosquitoes biting a human was drawn from a Poisson distribution $(\lambda=2.1)$ assuming a daily biting probability of 0.7 (ref. ${ }^{95}$ ) and a relative mosquito density of 3 (ref. ${ }^{94}$ ).

Human-to-mosquito transmission. Probabilities of human-to-mosquito ZIKV transmission were inferred from empirical data obtained with the mouse-to-mosquito transmission assay described above (Fig. S1). Mean transmission probabilities ( $\mu$ ) and their standard deviations (sd) were estimated from the infection rates of 171 mosquitoes tested by RT-PCR 14 days post infectious blood meal as described above. Transmission probabilities were used to derive a beta distribution of parameters $\alpha=((1-$ $\left.\mu) / s d^{2}-1 / \mu\right)^{*} \mu^{2}$ and $\beta=\alpha^{*}(1 / \mu-1)$ from which daily transmission probability for each simulated human was drawn until 6 days post infection. The parameters governing human-to-mosquito transmission were shared among all ZIKV strains.

Mosquito-to-human transmission. A 3-parameter logistic model was used to describe the cumulative proportion of infectious mosquitoes over time for each ZIKV strain using least-squares non-linear regression with the $d r m$ function in the $\mathrm{R}$ package $d r c^{96}$. The 3-parameter logistic function (L.3 equation in the $d r m$ function) is given by the formula: $f(x,(K, B, M))=K /(1+e-B($ time $-M))$ where $K$ represents the saturation level, $M$ represents the time at which transmission equals $50 \%$ of the saturation level $K$, and $B$ represents the maximum growth rate ${ }^{91}$. The probability of mosquito-to-human transmission for each contact between a human and an infected mosquito was determined by the predicted transmission probability inferred for each ZIKV strain. For each mosquito, an individual extrinsic incubation period (EIP) was drawn from the empirically determined logistic distribution of EIP values (mean $M$, scale $1 / B$ ). The EIP value was used as a threshold for the individual probability of virus transmission over time. Transmission probability was zero before the EIP, and it was randomly drawn from a binomial distribution of probability $K$ after the EIP. 
Implementation. Epidemiological simulations were run in R v3.6.0 using the packages foreach $^{97}$, doParalle/98 ${ }^{98}$ and nosoi ${ }^{56}$. Briefly, simulations were initiated with one infected human and zero infected mosquitoes and run for 400 days or until 100,000 humans or $1,000,000$ mosquitoes were reached, whichever occurred first. A total of 100 independent replicate simulations were run for each ZIKV strain.

\section{Acknowledgements}

We thank Catherine Lallemand for assistance with mosquito rearing, the Institut Pasteur animal facility staff for the breeding of Ifnar $1^{-/}$mice, and Anubis Vega-Rúa for initially providing the mosquito colony from Guadeloupe. We are grateful to the volunteers and the ICAReB staff for the human blood supply. We thank Elke Maas, Carolien De Keyzer and Lindsey Bervoets for technical assistance with the AG129 mouse experiments, the KU Leuven Rega animal facility staff for breeding the AG129 mice, and Jelle Matthijnssens and Daan Jansen for resequencing the ZIKV strains at the KU Leuven Rega Institute. We thank Alexandre Hego and the GIGA-Imaging platform for their precious help and advice, and Christian Alfano and Ivan Gladwyn- $\mathrm{Ng}$ for their guidance with the vertical transmission experiments.

\section{Funding}

This work was primarily funded by the European Union's Horizon 2020 research and innovation programme under ZikaPLAN grant agreement no. 734584 (to LL and JN) and under ZIKAlliance grant agreement no. 734548 (to LN and JN). This work was also supported by Agence Nationale de la Recherche (grants ANR-16-CE35-0004-01, ANR-17-ERC2-0016-01 and ANR-18-CE35-0003-01 to LL), the French Government's Investissement d'Avenir program Laboratoire d'Excellence Integrative Biology of Emerging Infectious Diseases (grant ANR-10-LABX-62-IBEID to LL and XM), the Inception program (Investissement d'Avenir grant ANR-16-CONV-0005 to LL), the Research Foundation Flanders (FWO PhD fellowship 1S21918N to SJ), and the Fonds de la Recherche Scientifique (FRIA PhD fellowship to MD). The funders had no role in study design, data collection and interpretation, or the decision to submit the work for publication. 


\section{Author contributions}

SJFK and LL designed and coordinated the study. FA, NJ, EFM, SD, CM and $A B$ carried out the mosquito experiments. SL and AF conducted the epidemiological modeling. SJ, LD and SJFK designed and performed the AG129 mouse experiments. $M D$ and $M G$ designed and performed the vertical transmission experiments. SD, SL, $F G$ and $E S-L$ performed the virus sequencing. $F A$ and $A B$ performed the phylogenetic analyses. CMR-V conducted the field collections to initiate the mosquito colony from Colombia. V-MC-L, RGJ, CTD, OumF, OusF and AAS obtained the virus isolates and organized their transfer. FA, CM, XM and LL designed and implemented the mouseto-mosquito transmission assay. JN supervised the AG129 mouse experiments. LN supervised the vertical transmission experiments. FA, SJ, MD, SJFK and LL prepared the figures. FA and LL analyzed the data and wrote the manuscript with input from all other authors. 


\section{References}

1 Boyer, S., Calvez, E., Chouin-Carneiro, T., Diallo, D. \& Failloux, A. B. An overview of mosquito vectors of Zika virus. Microbes Infect 20, 646-660, doi:10.1016/j.micinf.2018.01.006 (2018).

2 Gutierrez-Bugallo, G. et al. Vector-borne transmission and evolution of Zika virus. Nat Ecol Evol 3, 561-569, doi:10.1038/s41559-019-0836-z (2019).

3 Dick, G. W., Kitchen, S. F. \& Haddow, A. J. Zika virus. I. Isolations and serological specificity. Trans $R$ Soc Trop Med Hyg 46, 509-520, doi:10.1016/0035-9203(52)90042-4 (1952).

4 Musso, D. \& Gubler, D. J. Zika Virus. Clin Microbiol Rev 29, 487-524, doi:10.1128/CMR.00072-15 (2016).

5 Kindhauser, M. K., Allen, T., Frank, V., Santhana, R. S. \& Dye, C. Zika: the origin and spread of a mosquito-borne virus. Bull World Health Organ 94, 675686C, doi:10.2471/BLT.16.171082 (2016).

6 Duffy, M. R. et al. Zika virus outbreak on Yap Island, Federated States of Micronesia. N Engl J Med 360, 2536-2543, doi:10.1056/NEJMoa0805715 (2009).

7 Cao-Lormeau, V. M. et al. Zika virus, French polynesia, South pacific, 2013. Emerg Infect Dis 20, 1085-1086, doi:10.3201/eid2006.140138 (2014).

8 Cao-Lormeau, V. M. \& Musso, D. Emerging arboviruses in the Pacific. Lancet 384, 1571-1572, doi:10.1016/S0140-6736(14)61977-2 (2014).

9 Pan American Health Organization / World Health Organization, Zika Cumulative Cases. Cited 31 May 2018.

10 Cauchemez, S. et al. Association between Zika virus and microcephaly in French Polynesia, 2013-15: a retrospective study. Lancet 387, 2125-2132, doi:10.1016/S0140-6736(16)00651-6 (2016).

11 Oehler, E. et al. Zika virus infection complicated by Guillain-Barre syndrome-case report, French Polynesia, December 2013. Euro Surveill 19, doi:10.2807/1560-7917.es2014.19.9.20720 (2014).

12 World Health Organization. Countries and territories with current or previous Zika virus transmission. Updated July 2019. 
13 Haddow, A. D. et al. Genetic characterization of Zika virus strains: geographic expansion of the Asian lineage. PLoS Negl Trop Dis 6, e1477, doi:10.1371/journal.pntd.0001477 (2012).

14 Faria, N. R. et al. Zika virus in the Americas: Early epidemiological and genetic findings. Science 352, 345-349, doi:10.1126/science.aaf5036 (2016).

15 Duong, V. et al. Low Circulation of Zika Virus, Cambodia, 2007-2016. Emerg Infect Dis 23, 296-299, doi:10.3201/eid2302.161432 (2017).

16 Ruchusatsawat, K. et al. Long-term circulation of Zika virus in Thailand: an observational study. Lancet Infect Dis 19, 439-446, doi:10.1016/S14733099(18)30718-7 (2019).

17 Maurer-Stroh, S. et al. South-east Asian Zika virus strain linked to cluster of cases in Singapore, August 2016. Euro Surveill 21, doi:10.2807/15607917.ES.2016.21.38.30347 (2016).

18 Chu, D. T., Ngoc, V. T. N. \& Tao, Y. Zika virus infection in Vietnam: current epidemic, strain origin, spreading risk, and perspective. Eur J Clin Microbiol Infect Dis 36, 2041-2042, doi:10.1007/s10096-017-3030-8 (2017).

19 Grubaugh, N. D., Ishtiaq, F., Setoh, Y. X. \& Ko, A. I. Misperceived Risks of Zikarelated Microcephaly in India. Trends Microbiol 27, 381-383, doi:10.1016/j.tim.2019.02.004 (2019).

20 Lan, P. T. et al. Fetal Zika Virus Infection in Vietnam. PLoS Curr 9, doi:10.1371/currents.outbreaks.1c8f631e0ef8cd7777d639eba48647fa (2017).

21 Moi, M. L. et al. Zika virus infection and microcephaly in Vietnam. Lancet Infect Dis 17, 805-806, doi:10.1016/S1473-3099(17)30412-7 (2017).

22 Kuadkitkan, A., Wikan, N., Sornjai, W. \& Smith, D. R. Zika virus and microcephaly in Southeast Asia: A cause for concern? J Infect Public Health 13, 11-15, doi:10.1016/j.jiph.2019.09.012 (2020).

23 Wongsurawat, T. et al. Case of Microcephaly after Congenital Infection with Asian Lineage Zika Virus, Thailand. Emerg Infect Dis 24, doi:10.3201/eid2409.180416 (2018).

24 Lourenco, J. et al. Epidemiology of the Zika Virus Outbreak in the Cabo Verde Islands, West Africa. PLoS Curr doi:10.1371/currents.outbreaks.19433b1e4d007451c691f138e1e67e8c (2018). 
25 Faye, O. et al. Genomic Epidemiology of 2015-2016 Zika Virus Outbreak in Cape Verde. Emerg Infect Dis 26, 1084-1090, doi:10.3201/eid2606.190928 (2020).

26 Hill, S. C. et al. Emergence of the Asian lineage of Zika virus in Angola: an outbreak investigation. Lancet Infect Dis 19, 1138-1147, doi:10.1016/S14733099(19)30293-2 (2019).

27 Musso, D., Ko, A. I. \& Baud, D. Zika Virus Infection - After the Pandemic. N Engl J Med 381, 1444-1457, doi:10.1056/NEJMra1808246 (2019).

28 Anfasa, F. et al. Phenotypic Differences between Asian and African Lineage Zika Viruses in Human Neural Progenitor Cells. mSphere 2, doi:10.1128/mSphere.00292-17 (2017).

29 Bowen, J. R. et al. Zika Virus Antagonizes Type I Interferon Responses during Infection of Human Dendritic Cells. PLoS Pathog 13, e1006164, doi:10.1371/journal.ppat.1006164 (2017).

30 Gabriel, E. et al. Recent Zika Virus Isolates Induce Premature Differentiation of Neural Progenitors in Human Brain Organoids. Cell Stem Cell 20, 397-406 e395, doi:10.1016/j.stem.2016.12.005 (2017).

31 Hamel, R. et al. African and Asian Zika virus strains differentially induce early antiviral responses in primary human astrocytes. Infect Genet Evol 49, 134-137, doi:10.1016/j.meegid.2017.01.015 (2017).

32 Sheridan, M. A. et al. Vulnerability of primitive human placental trophoblast to Zika virus. Proc Natl Acad Sci U S A 114, E1587-E1596, doi:10.1073/pnas.1616097114 (2017).

33 Simonin, Y. et al. Zika Virus Strains Potentially Display Different Infectious Profiles in Human Neural Cells. EBioMedicine 12, 161-169, doi:10.1016/j.ebiom.2016.09.020 (2016).

34 Smith, D. R. et al. African and Asian Zika Virus Isolates Display Phenotypic Differences Both In Vitro and In Vivo. Am J Trop Med Hyg 98, 432-444, doi:10.4269/ajtmh.17-0685 (2018).

35 Pompon, J. et al. A Zika virus from America is more efficiently transmitted than an Asian virus by Aedes aegypti mosquitoes from Asia. Sci Rep 7, 1215, doi:10.1038/s41598-017-01282-6 (2017). 
36 Weger-Lucarelli, J. et al. Vector Competence of American Mosquitoes for Three Strains of Zika Virus. PLoS Negl Trop Dis 10, e0005101, doi:10.1371/journal.pntd.0005101 (2016).

37 Liu, Z. et al. Competence of Aedes aegypti, Ae. albopictus, and Culex quinquefasciatus Mosquitoes as Zika Virus Vectors, China. Emerg Infect Dis 23, 1085-1091, doi:10.3201/eid2307.161528 (2017).

38 Roundy, C. M. et al. Variation in Aedes aegypti Mosquito Competence for Zika Virus Transmission. Emerg Infect Dis 23, 625-632, doi:10.3201/eid2304.161484 (2017).

39 Calvez, E. et al. Differential transmission of Asian and African Zika virus lineages by Aedes aegypti from New Caledonia. Emerg Microbes Infect 7, 159, doi:10.1038/s41426-018-0166-2 (2018).

40 Lazear, H. M. et al. A Mouse Model of Zika Virus Pathogenesis. Cell Host Microbe 19, 720-730, doi:10.1016/j.chom.2016.03.010 (2016).

41 Tripathi, S. et al. A novel Zika virus mouse model reveals strain specific differences in virus pathogenesis and host inflammatory immune responses. PLoS Pathog 13, e1006258, doi:10.1371/journal.ppat.1006258 (2017).

42 Widman, D. G. et al. A Reverse Genetics Platform That Spans the Zika Virus Family Tree. MBio 8, doi:10.1128/mBio.02014-16 (2017).

43 Dowall, S. D. et al. A Susceptible Mouse Model for Zika Virus Infection. PLoS Negl Trop Dis 10, e0004658, doi:10.1371/journal.pntd.0004658 (2016).

44 Shao, Q. et al. The African Zika virus MR-766 is more virulent and causes more severe brain damage than current Asian lineage and dengue virus. Development 144, 4114-4124, doi:10.1242/dev.156752 (2017).

45 Jaeger, A. S. et al. Zika viruses of African and Asian lineages cause fetal harm in a mouse model of vertical transmission. PLoS Negl Trop Dis 13, e0007343, doi:10.1371/journal.pntd.0007343 (2019).

46 Goodfellow, F. T. et al. Strain-Dependent Consequences of Zika Virus Infection and Differential Impact on Neural Development. Viruses 10, doi:10.3390/v10100550 (2018).

47 Willard, K. A. et al. Zika Virus Exhibits Lineage-Specific Phenotypes in Cell Culture, in Aedes aegypti Mosquitoes, and in an Embryo Model. Viruses 9, doi:10.3390/v9120383 (2017). 
48 Duggal, N. K. et al. Differential Neurovirulence of African and Asian Genotype Zika Virus Isolates in Outbred Immunocompetent Mice. Am J Trop Med Hyg 97, 1410-1417, doi:10.4269/ajtmh.17-0263 (2017).

49 Aliota, M. T. et al. Heterologous Protection against Asian Zika Virus Challenge in Rhesus Macaques. PLoS Negl Trop Dis 10, e0005168, doi:10.1371/journal.pntd.0005168 (2016).

50 Rayner, J. O. et al. Comparative Pathogenesis of Asian and African-Lineage Zika Virus in Indian Rhesus Macaque's and Development of a Non-Human Primate Model Suitable for the Evaluation of New Drugs and Vaccines. Viruses 10, doi:10.3390/v10050229 (2018).

51 Koide, F. et al. Development of a Zika Virus Infection Model in Cynomolgus Macaques. Front Microbiol 7, 2028, doi:10.3389/fmicb.2016.02028 (2016).

52 Cugola, F. R. et al. The Brazilian Zika virus strain causes birth defects in experimental models. Nature 534, 267-271, doi:10.1038/nature18296 (2016).

53 Liu, S., DeLalio, L. J., Isakson, B. E. \& Wang, T. T. AXL-Mediated Productive Infection of Human Endothelial Cells by Zika Virus. Circ Res 119, 1183-1189, doi:10.1161/CIRCRESAHA.116.309866 (2016).

54 Faye, O. et al. Molecular evolution of Zika virus during its emergence in the 20(th) century. PLoS Negl Trop Dis 8, e2636, doi:10.1371/journal.pntd.0002636 (2014).

55 Simonin, Y., van Riel, D., Van de Perre, P., Rockx, B. \& Salinas, S. Differential virulence between Asian and African lineages of Zika virus. PLoS Negl Trop Dis 11, e0005821, doi:10.1371/journal.pntd.0005821 (2017).

56 Lequime, S., Bastide, P., Dellicour, S., Lemey, P. \& Baele, G. nosoi: A stochastic agent-based transmission chain simulation framework in R. Methods Ecol Evol, doi:10.1111/2041-210X.13422 (2020).

57 Zmurko, J. et al. The Viral Polymerase Inhibitor 7-Deaza-2'-C-Methyladenosine Is a Potent Inhibitor of In Vitro Zika Virus Replication and Delays Disease Progression in a Robust Mouse Infection Model. PLoS Negl Trop Dis 10, e0004695, doi:10.1371/journal.pntd.0004695 (2016).

58 Gladwyn-Ng, I. et al. Stress-induced unfolded protein response contributes to Zika virus-associated microcephaly. Nat Neurosci 21, 63-71, doi:10.1038/s41593-017-0038-4 (2018). 
59 Zhang, W. et al. In utero infection of Zika virus leads to abnormal central nervous system development in mice. Sci Rep 9, 7298, doi:10.1038/s41598-019-433036 (2019).

60 Weaver, S. C. Emergence of Epidemic Zika Virus Transmission and Congenital Zika Syndrome: Are Recently Evolved Traits to Blame? mBio 8, doi:10.1128/mBio.02063-16 (2017).

61 Haby, M. M., Pinart, M., Elias, V. \& Reveiz, L. Prevalence of asymptomatic Zika virus infection: a systematic review. Bull World Health Organ 96, 402-413D, doi:10.2471/BLT.17.201541 (2018).

62 Herrera, B. B. et al. Continued Transmission of Zika Virus in Humans in West Africa, 1992-2016. J Infect Dis 215, 1546-1550, doi:10.1093/infdis/jix182 (2017).

63 Kayiwa, J. T. et al. Confirmation of Zika virus infection through hospital-based sentinel surveillance of acute febrile illness in Uganda, \&nbsp;2014-2017. J Gen Virol 99, 1248-1252, doi:10.1099/jgv.0.001113 (2018).

64 Kisuya, B., Masika, M. M., Bahizire, E. \& Oyugi, J. O. Seroprevalence of Zika virus in selected regions in Kenya. Trans $R$ Soc Trop Med Hyg 113, 735-739, doi:10.1093/trstmh/trz077 (2019).

65 Mathe, P. et al. Low Zika virus seroprevalence among pregnant women in North Central Nigeria, 2016. J Clin Virol 105, 35-40, doi:10.1016/j.jcv.2018.05.011 (2018).

66 Nurtop, E. et al. A Report of Zika Virus Seroprevalence in Republic of the Congo. Vector Borne Zoonotic Dis 20, 40-42, doi:10.1089/vbz.2019.2466 (2020).

67 L'Azou, M. et al. Dengue seroprevalence: data from the clinical development of a tetravalent dengue vaccine in 14 countries (2005-2014). Trans $R$ Soc Trop Med Hyg 112, 158-168, doi:10.1093/trstmh/try037 (2018).

68 Gake, B. et al. Low seroprevalence of Zika virus in Cameroonian blood donors. Braz J Infect Dis 21, 481-483, doi:10.1016/j.bjid.2017.03.018 (2017).

69 Buchwald, A. G., Hayden, M. H., Dadzie, S. K., Paull, S. H. \& Carlton, E. J. Aedes-borne disease outbreaks in West Africa: A call for enhanced surveillance. Acta Trop 209, 105468, doi:10.1016/j.actatropica.2020.105468 (2020). 
70 Garske, T. et al. Yellow Fever in Africa: estimating the burden of disease and impact of mass vaccination from outbreak and serological data. PLoS Med 11, e1001638, doi:10.1371/journal.pmed.1001638 (2014).

71 Aubry, F. et al. www.biorxiv.org/content/early/2018/06/10/342741 (bioRxiv 2018).

72 Gloria-Soria, A. et al. Global genetic diversity of Aedes aegypti. Mol Ecol 25, 5377-5395, doi:10.1111/mec.13866 (2016).

73 Kotsakiozi, P. et al. Population structure of a vector of human diseases: Aedes aegypti in its ancestral range, Africa. Ecol Evol 8, 7835-7848, doi:10.1002/ece3.4278 (2018).

74 Sassetti, M. et al. First case of confirmed congenital Zika syndrome in continental Africa. Trans $R$ Soc Trop Med Hyg 112, 458-462, doi:10.1093/trstmh/try074 (2018).

75 Rosenstierne, M. W. et al. Zika Virus IgG in Infants with Microcephaly, GuineaBissau, 2016. Emerg Infect Dis 24, 948-950, doi:10.3201/eid2405.180153 (2018).

76 Vermillion, M. S. et al. Intrauterine Zika virus infection of pregnant immunocompetent mice models transplacental transmission and adverse perinatal outcomes. Nat Commun 8, 14575, doi:10.1038/ncomms14575 (2017).

77 Baidaliuk, A. et al. Cell-Fusing Agent Virus Reduces Arbovirus Dissemination in Aedes aegypti Mosquitoes In Vivo. J Virol 93, doi:10.1128/JVI.00705-19 (2019).

78 Fontaine, A., Jiolle, D., Moltini-Conclois, I., Lequime, S. \& Lambrechts, L. Excretion of dengue virus RNA by Aedes aegypti allows non-destructive monitoring of viral dissemination in individual mosquitoes. Sci Rep 6, 24885, doi:10.1038/srep24885 (2016).

79 Lequime, S., Richard, V., Cao-Lormeau, V. M. \& Lambrechts, L. Full-genome dengue virus sequencing in mosquito saliva shows lack of convergent positive selection during transmission by Aedes aegypti. Virus Evol 3, vex031, doi:10.1093/ve/vex031 (2017).

80 Bolger, A. M., Lohse, M. \& Usadel, B. Trimmomatic: a flexible trimmer for Illumina sequence data. Bioinformatics 30, 2114-2120, doi:10.1093/bioinformatics/btu170 (2014).

81 Langmead, B. \& Salzberg, S. L. Fast gapped-read alignment with Bowtie 2. Nat Methods 9, 357-359, doi:10.1038/nmeth.1923 (2012). 
82 Boisvert, S., Laviolette, F. \& Corbeil, J. Ray: simultaneous assembly of reads from a mix of high-throughput sequencing technologies. J Comput Biol 17, 1519-1533, doi:10.1089/cmb.2009.0238 (2010).

83 Nurk, S., Meleshko, D., Korobeynikov, A. \& Pevzner, P. A. metaSPAdes: a new versatile metagenomic assembler. Genome Res 27, 824-834, doi:10.1101/gr.213959.116 (2017).

84 Altschul, S. F., Gish, W., Miller, W., Myers, E. W. \& Lipman, D. J. Basic local alignment search tool. J Mol Biol 215, 403-410, doi:10.1016/S00222836(05)80360-2 (1990).

85 Katoh, K. \& Standley, D. M. MAFFT multiple sequence alignment software version 7: improvements in performance and usability. Mol Biol Evol 30, 772780, doi:10.1093/molbev/mst010 (2013).

86 Kalyaanamoorthy, S., Minh, B. Q., Wong, T. K. F., von Haeseler, A. \& Jermiin, L. S. ModelFinder: fast model selection for accurate phylogenetic estimates. Nat Methods 14, 587-589, doi:10.1038/nmeth.4285 (2017).

87 Hoang, D. T., Chernomor, O., von Haeseler, A., Minh, B. Q. \& Vinh, L. S. UFBoot2: Improving the Ultrafast Bootstrap Approximation. Mol Biol Evol 35, 518-522, doi:10.1093/molbev/msx281 (2018).

88 Nguyen, L. T., Schmidt, H. A., von Haeseler, A. \& Minh, B. Q. IQ-TREE: a fast and effective stochastic algorithm for estimating maximum-likelihood phylogenies. Mol Biol Evol 32, 268-274, doi:10.1093/molbev/msu300 (2015).

89 FigTree, version 1.4.4, http://tree.bio.ed.ac.uk/software/figtree/ (2009).

90 Gong, Z., Xu, X. \& Han, G. Z. The Diversification of Zika Virus: Are There Two Distinct Lineages? Genome Biol Evol 9, 2940-2945, doi:10.1093/gbe/evx223 (2017).

91 Fontaine, A. et al. Epidemiological significance of dengue virus genetic variation in mosquito infection dynamics. PLoS Pathog 14, e1007187, doi:10.1371/journal.ppat.1007187 (2018).

92 Yakob, L., Kucharski, A., Hue, S. \& Edmunds, W. J. Low risk of a sexuallytransmitted Zika virus outbreak. Lancet Infect Dis 16, 1100-1102, doi:10.1016/S1473-3099(16)30324-3 (2016).

93 Lequime, S. \& Lambrechts, L. Vertical transmission of arboviruses in mosquitoes: a historical perspective. Infect Genet Evol 28, 681-690, doi:10.1016/j.meegid.2014.07.025 (2014). 
94 Favier, C. et al. Influence of spatial heterogeneity on an emerging infectious disease: the case of dengue epidemics. Proc Biol Sci 272, 1171-1177, doi:10.1098/rspb.2004.3020 (2005).

95 Andraud, M., Hens, N., Marais, C. \& Beutels, P. Dynamic epidemiological models for dengue transmission: a systematic review of structural approaches. PLoS One 7, e49085, doi:10.1371/journal.pone.0049085 (2012).

96 Ritz, C., Baty, F., Streibig, J. C. \& Gerhard, D. Dose-Response Analysis Using R. PLoS One 10, e0146021, doi:10.1371/journal.pone.0146021 (2015).

97 foreach: Provides Foreach Looping Construct for R, https://cran.rproject.org/web/packages/foreach/foreach.pdf (2010).

98 doParallel: Foreach Parallel Adaptor for the 'parallel' Package, https://cran.rproject.org/web/packages/doParallel/doParallel.pdf (2019). 


\section{Supporting information}

Supplemental Table 1. ZIKV strains included in this study. The name of the ZIKV strains used in the study with their country of origin, original strain name, source of isolation, year of collection and the passage history prior to use in this study are indicated. TS: Toxorhynchites splendens mosquitoes; C6/36: Aedes albopictus cells; Vero: green monkey kidney cells; SM: suckling mouse brains.

\begin{tabular}{|c|c|c|c|c|c|}
\hline $\begin{array}{c}\text { Name in this } \\
\text { study }\end{array}$ & Country & Strain ID & Source & Year & $\begin{array}{c}\text { Passage } \\
\text { history }\end{array}$ \\
\hline F_Polynesia_2013 & $\begin{array}{c}\text { French } \\
\text { Polynesia }\end{array}$ & PF13/251013-18 & Human serum & 2013 & C6/36-4 \\
\hline Puerto_Rico_2015 & $\begin{array}{c}\text { Puerto } \\
\text { Rico }\end{array}$ & PRVABC59 & Human serum & 2015 & Vero-2; C6/36-3 \\
\hline Philippines_2012 & Philippines & CPC-0740 & Human serum & 2012 & $\begin{array}{c}\text { TS-1; C6/36-2; } \\
\text { Vero-1; C6/36-1 }\end{array}$ \\
\hline Thailand_2014 & Thailand & SV0127-14 & Human serum & 2014 & $\begin{array}{c}\text { TS-1; C6/36-2; } \\
\text { Vero-1; C6/36-1 }\end{array}$ \\
\hline Cambodia_2010 & Cambodia & FSS13025 & Human serum & 2010 & $\begin{array}{c}\text { Vero 2; SM-1; } \\
\text { C6/36-1 }\end{array}$ \\
\hline Senegal_2011 & Senegal & Kedougou2011 & $\begin{array}{c}\text { Pool of Aedes spp. } \\
\text { and Mansonia spp. }\end{array}$ & 2011 & C6/36-2 \\
\hline Senegal_2015 & Senegal & Kedougou2015 & $\begin{array}{c}\text { Pool of Aedes spp. } \\
\text { and Mansonia spp. }\end{array}$ & 2015 & C6/36-2 \\
\hline
\end{tabular}




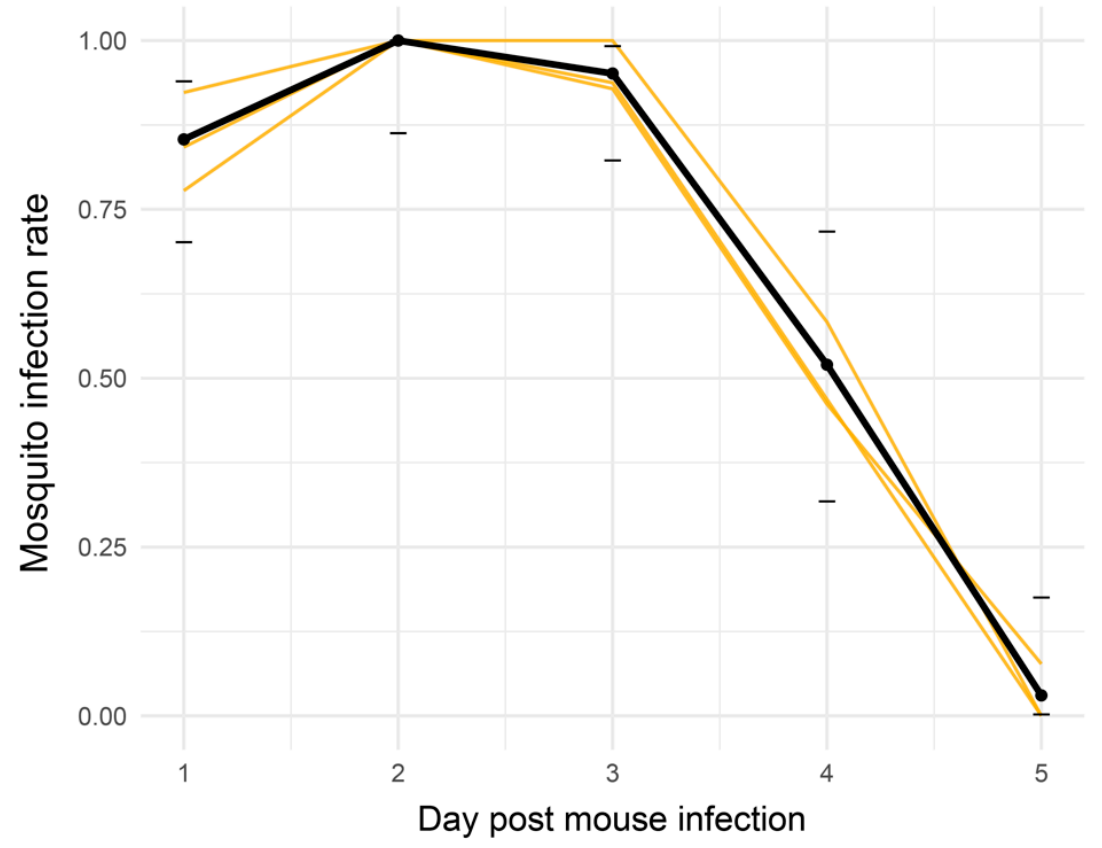

Supplemental Fig. 1. Mouse-to-mosquito ZIKV transmission probability over time. The lines represent the proportion of mosquitoes (median 13 females per time point and per mouse; range 0-19) that tested ZIKV-positive 14 days after taking a blood meal on an Ifnar $1^{-/}$mouse ( $\mathrm{n}=3$ mice) inoculated with $10^{5} \mathrm{FFU}$ of the Cambodia_2010 ZIKV strain on day 0 . Gold lines represent the transmission probabilities for the 3 individual mice during their viremic period and the black line represent their mean. Horizontal ticks indicate the $95 \%$ confidence intervals of the probabilities. 


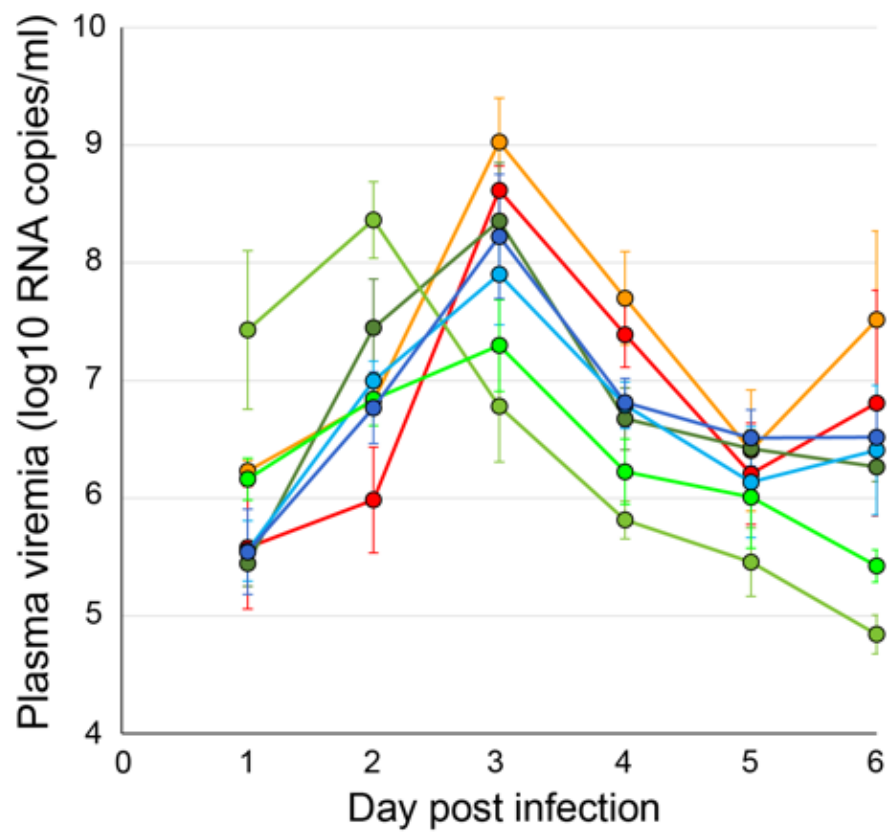

\section{Asian lineage}

○ F_Polynesia_2013

- Puerto Rico_2015

Thailand_2014

O Philippines_2012

- Cambodia_2010

\section{African lineage}

- Senegal_2015

○ Senegal_2011

\section{Supplemental Fig. 2. Variation in viremia kinetics between ZIKV strains after} inoculation of a high virus dose. Time course of ZIKV viremia in AG129 mice inoculated on day 0 with $10^{3}$ PFU of ZIKV ( $n=8-10$ mice per strain). Viremia is expressed in $\log _{10}$-transformed viral genome copies per $\mathrm{ml}$ of plasma (mean \pm standard error). 


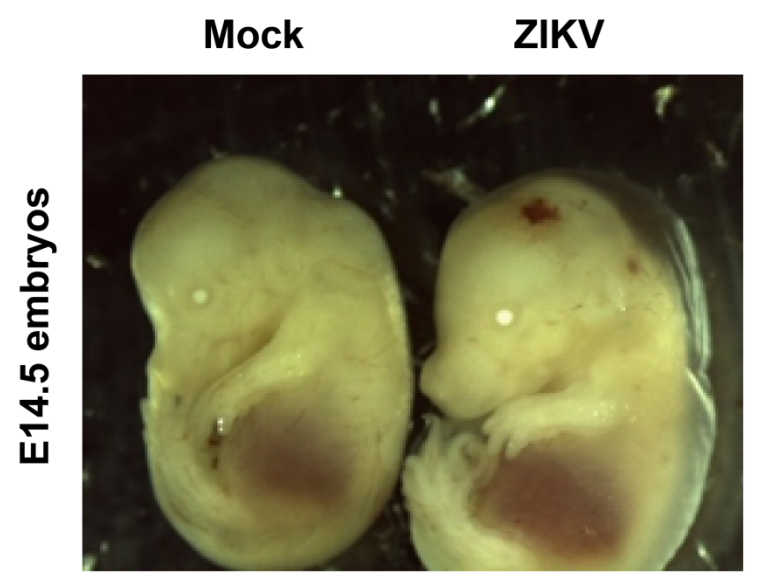

Supplemental Fig. 3. Intraplacental injections of ZIKV induce subcutaneous edema. Photos show representative E14.5 embryos after intraplacental injection of mock inoculum (left; $n=10$ embryos) or ZIKV Senegal_2015 strain (right; n=9 embryos) at E10.5. 

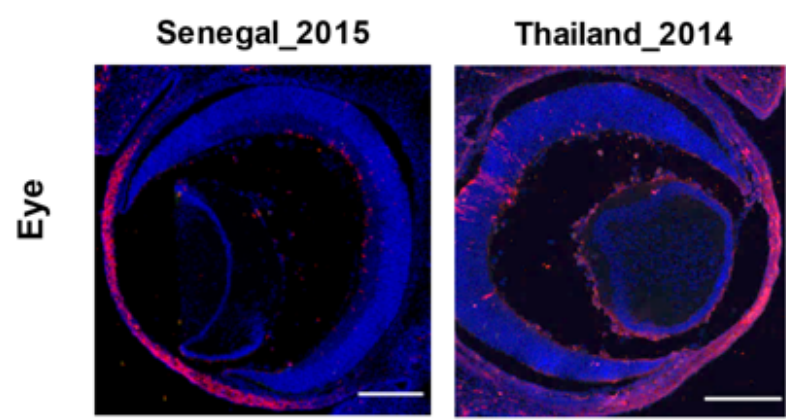

F_Polynesia_2013

Mock
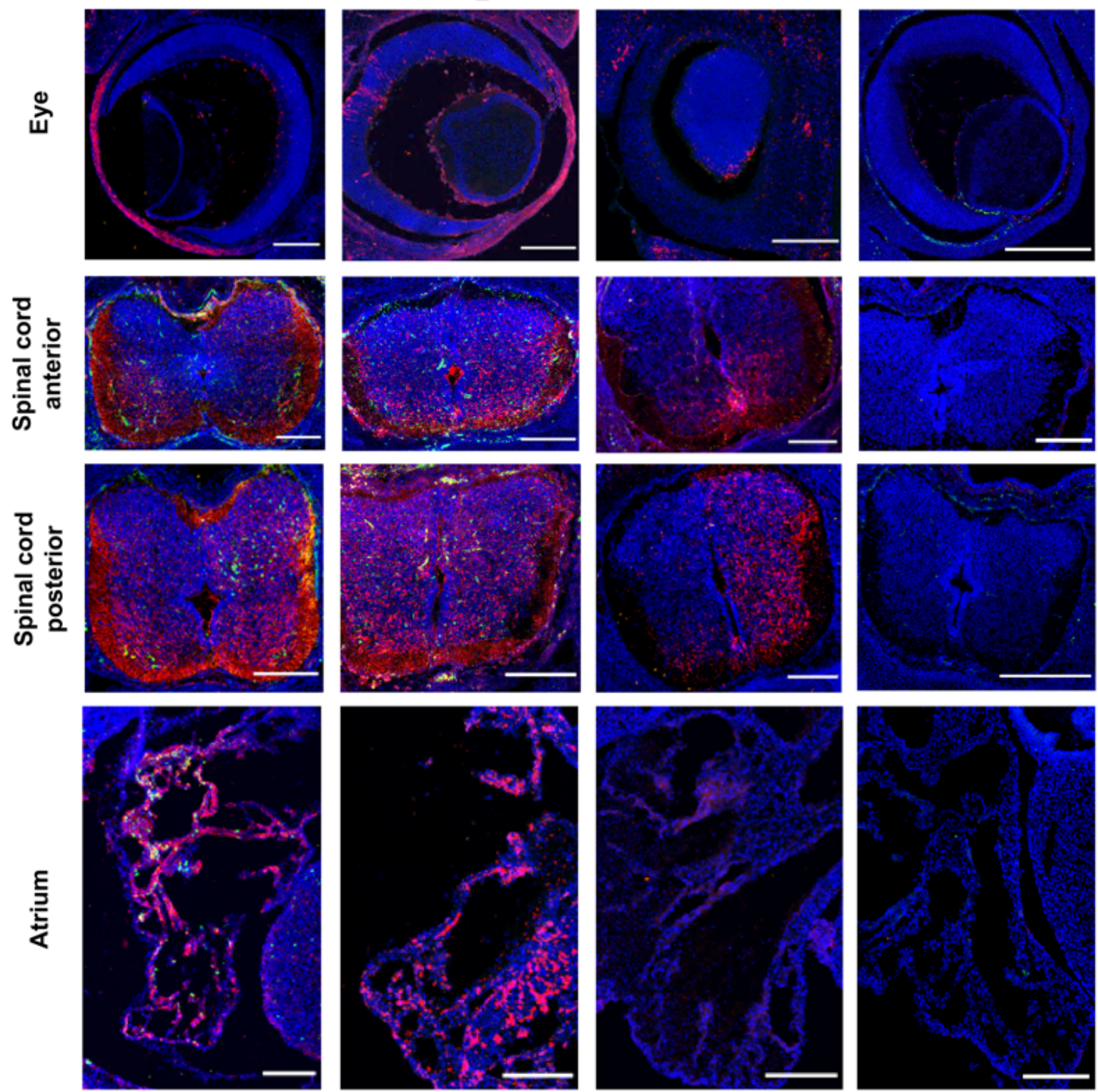

Supplemental Fig. 4. African and Asian ZIKV strains show comparable organs tropism. Immunocompetent mouse embryos were infected at E10.5 by intraplacental injection of 500-1,000 PFU of ZIKV and examined at E14.5 by microdissection. Immunolabeling of embryonic eye, anterior and posterior spinal cord and atrium sections representative of each ZIKV strain tested ( $n=3$ embryos per virus strain). Blue, green and red colors indicate DAPI, anti-cleaved caspase 3 (ACC3) and ZIKV stainings, respectively. The scale bars represent $200 \mu \mathrm{m}$. 